\title{
Front Matter: Volume 7102
}

, "Front Matter: Volume 7102," Proc. SPIE 7102, Optical Fabrication, Testing, and Metrology III, 710201 (15 October 2008); doi: 10.1117/12.817248

SPIE. Event: Optical Systems Design, 2008, Glasgow, Scotland, United Kingdom 


\title{
PROCEEDINGS OF SPIE
}

\section{Optical Fabrication, Testing, and Metrology III}

\author{
Angela Duparré \\ Roland Geyl \\ Editors \\ 2-4 September 2008 \\ Glasgow, United Kingdom \\ Sponsored by \\ SPIE Europe \\ Cosponsored by \\ Scottish Optoelectronic Association (United Kingdom) \\ Cooperating Organisations \\ EFDS-Europäische Forschungsgesellschaft Dünne Schichten e.V. (Germany) \\ Institute of Photonics (United Kingdom) \\ OpTIC Technium (United Kingdom) \\ WOF-Welsh Optoelectronics Forum (United Kingdom) \\ Published by \\ SPIE
}

Volume 7102 
The papers included in this volume were part of the technical conference cited on the cover and title page. Papers were selected and subject to review by the editors and conference program committee. Some conference presentations may not be available for publication. The papers published in these proceedings reflect the work and thoughts of the authors and are published herein as submitted. The publisher is not responsible for the validity of the information or for any outcomes resulting from reliance thereon.

Please use the following format to cite material from this book:

Author(s), "Title of Paper," in Optical Fabrication, Testing, and Metrology III, edited by Angela Duparré, Roland Geyl, Proceedings of SPIE Vol. 7102 (SPIE, Bellingham, WA, 2008) Article CID Number.

ISSN 0277-786X

ISBN 9780819473325

Published by

SPIE

P.O. Box 10, Bellingham, Washington 98227-0010 USA

Telephone +1 3606763290 (Pacific Time) · Fax +1 3606471445

SPIE.org

Copyright (C 2008, Society of Photo-Optical Instrumentation Engineers

Copying of material in this book for internal or personal use, or for the internal or personal use of specific clients, beyond the fair use provisions granted by the U.S. Copyright Law is authorized by SPIE subject to payment of copying fees. The Transactional Reporting Service base fee for this volume is $\$ 18.00$ per article (or portion thereof), which should be paid directly to the Copyright Clearance Center (CCC), 222 Rosewood Drive, Danvers, MA 01923. Payment may also be made electronically through CCC Online at copyright.com. Other copying for republication, resale, advertising or promotion, or any form of systematic or multiple reproduction of any material in this book is prohibited except with permission in writing from the publisher. The CCC fee code is $0277-786 \mathrm{X} / 08 / \$ 18.00$.

Printed in the United States of America.

Publication of record for individual papers is online in the SPIE Digital Library.

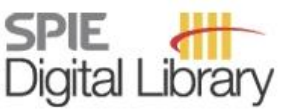

SPIEDigitallibrary.org

Paper Numbering: Proceedings of SPIE follow an e-First publication model, with papers published first online and then in print and on CD-ROM. Papers are published as they are submitted and meet publication criteria. A unique, consistent, permanent citation identifier (CID) number is assigned to each article at the time of the first publication. Utilization of CIDs allows articles to be fully citable as soon they are published online, and connects the same identifier to all online, print, and electronic versions of the publication. SPIE uses a six-digit CID article numbering system in which:

- The first four digits correspond to the SPIE volume number.

- The last two digits indicate publication order within the volume using a Base 36 numbering system employing both numerals and letters. These two-number sets start with 00, 01, 02, 03, 04, 05, $06,07,08,09,0 A, 0 B \ldots .0 Z$, followed by $10-1 Z, 20-2 Z$, etc.

The CID number appears on each page of the manuscript. The complete citation is used on the first page, and an abbreviated version on subsequent pages. Numbers in the index correspond to the last two digits of the six-digit CID number. 


\title{
Contents
}

\author{
ix Conference Committee \\ xi Optical system design reliance on technology development (Plenary Presentation) \\ I. A. Neil, ScotOptix (Switzerland) \\ xxxiii A perspective on the design of head-worn displays (Plenary Presentation) \\ J. Rolland, O. Cakmakci, F. Fournier, S. Vo, CREOL, The College of Optics and Photonics, \\ Univ. of Central Florida (United States)
}

\section{SESSION 1 NANO- AND MICROSTRUCTURES AND ELEMENTS}

710202 Nanostructured optical fibre for surface-enhanced Raman scattering sensing (Invited Paper) [7102-01]

D. J. White, A. P. Mazzolini, P. R. Stoddart, Swinburne Univ. of Technology (Australia)

710203 Localized measurements of optical thickness variations in femtosecond trimmed structures [7102-02]

A. L. Lereu, F. Lemarchand, M. Lequime, Institut Fresnel, CNRS (France)

710204 Phase and amplitude interferometric characterization of infrared nanostructured gratings [7102-03]

B. Toulon, ONERA (France); G. Vincent, ONERA (France) and LPN, CNRS (France); R. Haïdar, ONERA (France); S. Collin, LPN, CNRS (France); N. Guérineau, ONERA (France);

J.-L. Pelouard, LPN, CNRS (France); J. Primot, ONERA (France)

710205 Tunable liquid microlens array driven by pyroelectric effect: full interferometric characterization [7102-04]

L. Miccio, S. Grilli, V. Vespini, P. Ferraro, Istituto Nazionale di Ottica Applicata, CNR (Italy)

710206 Computer generated holograms: fabrication and application for precision optical testing [7102-05]

A. G. Poleshchuk, V. P. Korolkov, R. K. Nasyrov, Institute of Automation and Electrometry (Russia); J.-M. Asfour, Dioptic GmbH (Germany)

710207 Development of a goniometric light scatter instrument with sample imaging ability (Invited Paper) [7102-06]

M. Zerrad, M. Lequime, C. Deumié, C. Amra, Institut Fresnel, CNRS (France)

710208 Dynamic x-ray lithography for blazed diffractive optics fabrication [7102-07]

V. F. Pindyurin, B. G. Goldenberg, E. V. Petrova, U. V. Ancharova, V. S. Eliseev, Budker Institute of Nuclear Physics (Russia); V. P. Korolkov, R. K. Nasyrov, Institute of Automation and Electrometry (Russia) 
710209 Profilometric characterization of DOEs with continuous microrelief [7102-09]

V. P. Korolkov, S. V. Ostapenko, R. V. Shimansky, Institute of Automation and Electrometry (Russia)

\section{SESSION $2 \quad$ ASPHERIC AND COMPLEX SURFACES}

7102 OA Design for test and manufacture of complex multi-component optical instruments (Invited Paper) [7102-10]

S. Rolt, C. M. Dubbeldam, D. J. Robertson, D. A. Ryder, Univ. of Durham (United Kingdom)

$7102 \mathrm{OB} \quad$ Surface quality of a $1 \mathrm{~m}$ Zerodur part using an effective grinding mode [7102-11] X. Tonnellier, P. Shore, P. Morantz, D. Orton, Optic Technium (United Kingdom) and Cranfield Univ. (United Kingdom)

7102 OC Metal mirrors with excellent figure and roughness [7102-12]

R. Steinkopf, A. Gebhardt, S. Scheiding, M. Rohde, O. Stenzel, S. Gliech, Fraunhofer-Institut für Angewandte Optik und Feinmechanik (Germany); V. Giggel, H. Löscher, G. Ullrich, Carl Zeiss Jena GmbH (Germany); P. Rucks, Carl Zeiss AG (Germany); A. Duparre, S. Risse, R. Eberhardt, A. Tünnermann, Fraunhofer-Institut für Angewandte Optik und Feinmechanik (Germany)

7102 OD Aspherical manufacturing in terms of accuracy, efficiency, and surface forms based on practical experiences [7102-13]

S. R. Kiontke, asphericon GmbH (Germany); R. Steinkopf, Fraunhofer-Institut für Angewandte Optik und Feinmechanik (Germany)

$7102 \mathrm{OE} \quad$ Ion beam manufacturing of a graded-phase mirror for the generation of square "top hat" laser beams (Invited Paper) [7102-14]

R. Mercier, M. Lamare, M. Mullot, Institut d'Optique (France); V. Bagnoud, Gesellschaft für Schwerionenforschung Darmstadt GmbH (Germany); X. Ribeyre, CELIA, Univ. Bordeaux I (France); J. Luce, J. Néauport, CEA Cesta (France)

7102 OF Finish assessment of complex surfaces by advanced light scattering techniques [7102-15] S. Schröder, A. Duparré, Fraunhofer-Institut für Angewandte Optik und Feinmechanik (Germany)

7102 OG Applications and benefits of "perfectly bad" optical surfaces [7102-16] P. R. Dumas, R. W. Hallock, A. Pisarski, QED Technologies, Inc. (United States)

$7102 \mathrm{OH} \quad$ Modeling of the polishing process for aspheric optics [7102-17]

A. Kelm, R. Boerret, HTW Aalen (Germany); S. Sinzinger, TU Ilmenau (Germany)

7102 Ol Wave front related surface form tolerances for manufacturing higher grade optical components [7102-18] O. Falkenstörfer, Th. Herrmann, U. Krüger, S. Müller-Pfeiffer, JENOPTIK Laser, Optik, Systeme GmbH (Germany) 
SESSION $3 \quad$ INVITED SESSION: STANDARDS, ACCURACY, AND LIMITATIONS

7102 0J Laser beam homogenizing: limitations and constraints (Invited Paper) [7102-19]

R. Voelkel, K. J. Weible, SUSS MicroOptics SA (Switzerland)

7102 OK Digital cameras: optical footprints in a performance metric (Invited Paper) [7102-20]

L. Baker, Consultant (United Kingdom)

7102 OL Optical glasses and optical elements: comparison of specification standards ISO DIS 12123 and ISO 10110 (Invited Paper) [7102-21]

P. Hartmann, R. Jedamzik, SCHOTT AG (Germany)

7102 OM Optical roughness measurements on specially designed roughness standards (Invited Paper) [7102-22]

R. Danzl, F. Helmli, Alicona Imaging GmbH (Austria); P. Rubert, Rubert + Co. Ltd. (United

Kingdom); M. Prantl, Alicona Imaging GmbH (Austria)

\section{SESSION 4 SYSTEMS AND COMPONENTS I}

$7102 \mathrm{ON} \quad$ Model and simulation of fringe projection measurements as part of an assistance system for multi-component fringe projection sensors (Invited Paper) [7102-23]

A. Weckenmann, W. Hartmann, J. Weickmann, Univ. Erlangen-Nuremberg (Germany)

$710200 \quad$ Assembly and integration of optical systems for space applications [7102-24]

S. Blum, L. Francou, A. Herren, E. Rugi Grond, M. Siegrist, Oerlikon Space AG (Switzerland)

7102 OP Experience using a double pass Shack-Hartmann set-up on a DUV high NA high performance lens [7102-25]

M. Augustin, S. Müller-Pfeiffer, L. Körner, O. Falkenstörfer, Jenoptik Laser Optik Systeme GmbH (Germany)

$71020 Q \quad$ Intraocular lens characterization using a quadric-wave lateral shearing interferometer wave front sensor [7102-26]

W. Boucher, S. Velghe, B. Wattellier, PHASICS S.A. (France); D. Gatinel, Rothschild Foundation, CEROC (France)

7102 OR Test images of a sector star versus radial and axial merit functions [7102-27]

R. Hild, HTWK-Leipzig (Germany); J. Campos, M. Yzuel, Univ. Autònoma de Barcelona (Spain); C. Iemmi, Univ. de Buenos Aires (Argentina); J. C. Escalera, Univ. Autònoma de Barcelona (Spain)

SESSION 5 MATERIAL AND THIN FILM PROPERTIES

7102 OS Automating spectral measurements (Invited Paper) [7102-28]

F. T. Goldstein, FTG Software Associates (United States)

7102 OT Characterization of high reflecting coatings and optical materials by direct absorption and cavity ring down measurements [7102-29]

Ch. Mühlig, G. Schmidl, J. Bergmann, W. Triebel, Institute of Photonic Technology

(Germany) 
7102 OU Characterizations of UV-Iaser damage on fused silica surfaces [7102-30]

P. Cormont, Commissariat à I'Energie Atomique, CESTA (France); S. Palmier, Institut Fresnel, CNRS (France); B. Bertussi, Commissariat à I'Energie Atomique, LR (France); R. Courchinoux, G. Gaborit, Commissariat à l'Energie Atomique, CESTA (France); L. Gallais, Institut Fresnel, CNRS (France); L. Lamaignère, Commissariat à l'Energie Atomique, CESTA (France);

P. Legros, Institut François Magendie, Univ. de Bordeaux II (France); J.-L. Rullier, H. Bercegol, Commissariat à l'Energie Atomique, CESTA (France)

7102 OV Ray tracing approach to refractive index measurement of prism samples in a vacuum cell [7102-31]

M. Vannoni, G. Molesini, CNR, Istituto Nazionale di Ottica Applicata (Italy)

7102 OW Dispersion and temperature dependence of thermo-optic coefficients of optical materials over their whole transparency range: vectorial formalism and application to $\mathrm{KTiOPO}_{4}$ [7102-58]

J. Mangin, G. Gadret, Institut Carnot de Bourgogne, CNRS, Univ. de Bourgogne (France);

G. Mennerat, CEA/CESTA (France)

\section{SESSION 6 SYSTEMS AND COMPONENTS II}

$71020 \mathrm{X}$ Interferometrical testing of parabolic mirrors with diffracted spherical wavefront (Invited Paper) [7102-32]

L. Li, Jabil Inc. (United States)

SESSION 7 SPECIAL TECHNIQUES

7102 OY Ultrafine measurements of the thermal shift of Fabry-Perot resonances [7102-34]

S. Michel, F. Lemarquis, M. Lequime, Institut Fresnel, CNRS (France)

$71020 Z$ Optical compensation of macroscopical surface curvature in rough surface scatterometry [7102-35]

C. Hahlweg, H. Rothe, Helmut Schmidt Univ. (Germany)

710210 Optical inspection of micro-electromechanical systems [7102-36]

I. A. Sokolov, M. A. Bryushinin, P. M. Karavaev, A.F. Ioffe Physical-Technical Institute (Russia);

S. H. Khan, K. T. V. Grattan, City Univ. (United Kingdom)

710211 Fourier transform evaluation and numerical modeling of fluid flow in a Laval nozzle [7102-37]

F. Rodríguez, LOMG (Spain); B. V. Dorrío, A. F. Doval, Univ. of Vigo (Spain)

$710212 \quad$ Vortex polarimetry [7102-39]

G. Dyankov, S.-Y. Chen, F.-S. Yang, Industrial Technology Research Institute (Taiwan)

POSTER SESSION

710214 Investigation of an automated cleaning system for LMJ coating sol-gel process [7102-44]

E. Lavastre, S. Fontaine, R. Bergez, P. Wender, P. Cormont, C. Pellegrini, CEA-CESTA

(France); L. Beaurain, P. Belleville, CEA-Le Ripault (France) 
710218 Polishing and testing of the $3.5 \mathrm{~m} \mathrm{SiC} \mathrm{M1} \mathrm{mirror} \mathrm{of} \mathrm{the} \mathrm{Herschel} \mathrm{space} \mathrm{observatory} \mathrm{of} \mathrm{ESA}$ [7102-49]

T. Korhonen, P. Keinanen, M. Pasanen, Opteon Oy (Finland); A. Sillanpaa, Tuorla Observatory, Univ. of Turku (Finland)

710219 Polishing and testing of the $1.5 \mathrm{~m} \mathrm{SiC} \mathrm{M1} \mathrm{mirror} \mathrm{of} \mathrm{the} \mathrm{ALADIN} \mathrm{instrument} \mathrm{on} \mathrm{the} \mathrm{ADM-}$ Aeolus satellite of ESA [7102-52]

T. Korhonen, P. Keinanen, M. Pasanen, Opteon Oy (Finland); A. Sillanpaa, Tuorla Observatory, Univ. of Turku (Finland)

7102 1B Error behaviour in differential phase-shifting algorithms [7102-56]

M. Miranda, B. V. Dorrío, Univ. of Vigo (Spain)

7102 1C Spectral measurement in reflection on steeply aspheric surfaces [7102-57]

H. Piombini, CEA, Le Ripault (France); S. Bruynooghe, Carl Zeiss AG (Germany); P. Voarino, QOL (France)

Author Index 
Downloaded From: https://www.spiedigitallibrary.org/conference-proceedings-of-spie on 26 Apr 2023

Terms of Use: https://www.spiedigitallibrary.org/terms-of-use 


\title{
Conference Committee
}

\author{
Symposium Chair
}

David M. Williamson, West Malvern (United Kingdom); NRCA Fellow, Nikon Research Corporation of America (USA)

Symposium Cochairs

Andreas Tünnermann, Fraunhofer-Institut für Angewandte Optik und Feinmechanik (Germany)

Jean-Louis Meyzonnette, Institut d'Optique, Ecole Supérieure d'Optique (France)

Conference Chairs

Angela Duparré, Fraunhofer-Institut für Angewandte Optik und

Feinmechanik (Germany)

Roland Geyl, SAGEM SA (France)

Program Committee

Lionel R. Baker, LRB Consulting Ltd. (United Kingdom)

Michael Bray, MB Optique SARL (France)

Geneviève Chabassier, Commissariat à l'Energie Atomique (France)

Sead Doric, Doric Lenses Inc. (Canada)

James Harvey, College of Optics \& Photonics, University of Central Florida (United States)

Jacques Mangin, Université de Bourgogne (France)

Raymond F. Mercier, Institut d'Optique (France)

Roger P. Netterfield, Commonwealth Scientific and Industrial Research Organisation (Australia)

Alexander Pagis, El-Op Electrooptics Industries Ltd. (Israel)

Joanna Schmit, Veeco Instruments Inc. (United States)

Hans J. Tiziani, Universität Stuttgart (Germany)

Theo T. Tschudi, Technische Universität Darmstadt (Germany)

Reinhard Völkel, SUSS MicroOptics SA (Switzerland)

Lingli Wang, Jos. Schneider Optische Werke GmbH (Germany) 


\section{Session Chairs}

1 Nano- and Microstructures and Elements

Angela Duparré, Fraunhofer-Institut für Angewandte Optik und

Feinmechanik (Germany)

2 Aspheric and Complex Surfaces

Roland Geyl, SAGEM SA (France)

3 Invited Session: Standards, Accuracy, and Limitations

Lionel R. Baker, LRB Consulting Ltd. (United Kingdom)

$4 \quad$ Systems and Components I

Raymond F. Mercier, Institut d'Optique (France)

$5 \quad$ Material and Thin Film Properties

Geneviève Chabassier, Commissariat à l'Energie Atomique (France)

6 Systems and Components II

Jacques Mangin, Université de Bourgogne (France)

7 Special Techniques

Jacques Mangin, Université de Bourgogne (France) 


\title{
Optical system design reliance on technology development
}

\author{
lain A. Neil
}

\author{
ScotOptix
}

Via Miravalle 25A, CH-6900 Massagno, Switzerland

$++41(0) 919500158$ (voice \& fax)

++41 (0)79398 5524 (mobile)

scotoptix@aol.com

SPIE Europe
Optical Systems Design

"Optical system design reliance on technology development"

ScotOptix

\section{WELCOME}

\section{Firstly, thanks goes to SPIE, the organizing committee, Chairs and Co-Chairs of the Conference for acceptance of this presentation}




\title{
INTRODUCTION
}

\author{
Before commencing with an outline of \\ the presentation an explanation of the \\ the definitions used throughout is given
}

\section{DEFINITIONS}

(1) Technology development is the progression over time of manufactured optical components:

- Materials $\approx$ optical substrates

- Coatings $\approx$ multi-layer thin films

- Surfaces $\approx$ optical surface profiles

2 Optical design software is a tool to apply technology

3 Optical designer 'creates' the optics portion of the of the optical system design utilizing optical design software to apply technology

(4) FOV is Field of View \& NA is Numerical Aperture 


\section{DEFINITIONS (Cont'd)}

(2) Object is to the left and Image is to

Wavelength $\lambda(\mathrm{nm})$ the right unless otherwise shown

6 Three wavebands discussed:

- Infrared $\approx 0.7-1.5,3-5 \&$ 8-13 $\mu \mathrm{m}$ (700-1500, 3000-5000 \& 8000-13000nm)

- Visible $\approx 0.435-0.656 \mu \mathrm{m}$

(435-656nm)

- Ultraviolet $\approx 0.434 \sim 0.013 \mu \mathrm{m}$

(434.4-13.4nm)

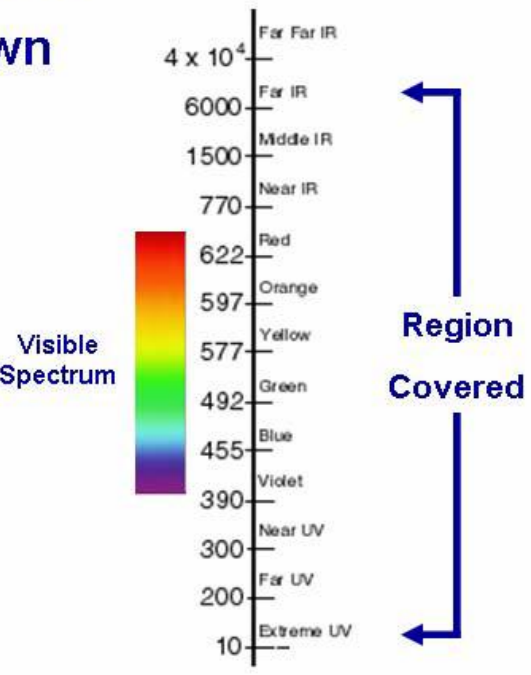

SPIE Europe

\section{OUTLINE}

- By way of mainly the US Patent database, examples are given to illustrate the reliance of optical system design on key technology

- The examples are categorized by waveband of operation and partly chronologically

- Performance characteristics are not discussed but all examples may be considered high performance for their intended applications 


\section{WAVEBAND 1 \\ INFRARED}

SPIE Europe

"Optical system design reliance on technology development"

\section{ScotOptix}

\section{EXAMPLE 1.1 \\ PETZVAL OBJECTIVE - SECURITY}

Passively Athermalized System $E F L=51 \mathrm{~mm} F / 1.5 \quad F O V \varnothing=5^{\circ}$ Waveband $=8-13 \mu \mathrm{m}$

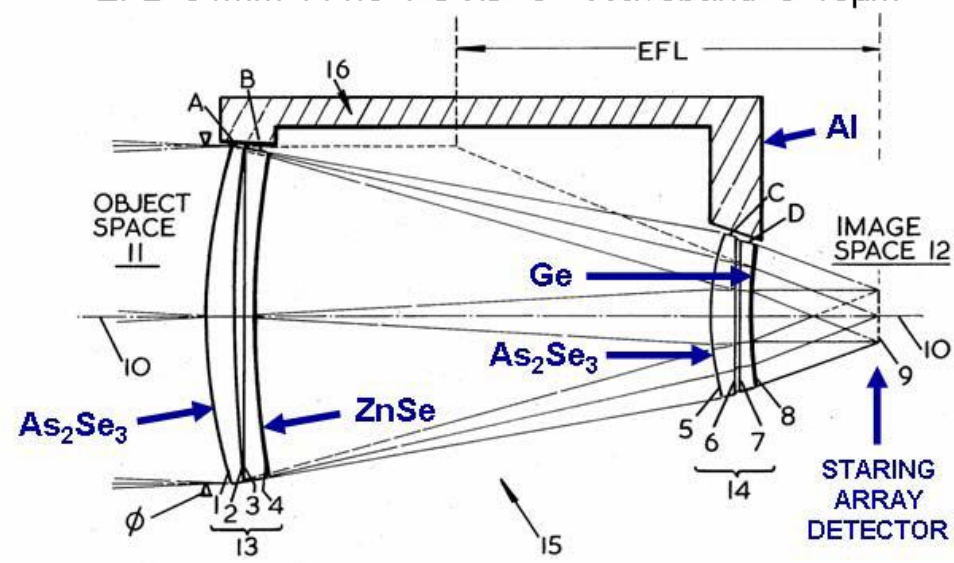

KEY TECHNOLOGY

\begin{tabular}{l|l|}
$\checkmark$ & MATERIAL \\
\hline
\end{tabular} COATING SURFACE BENEFITS SOLID STATE ROBUST ISSUES TOXIC MATERIAL MATERIAL QUALITY

US Pat. No. 4,505,535 A1 I.A.Neil Mar. 19, 1985 


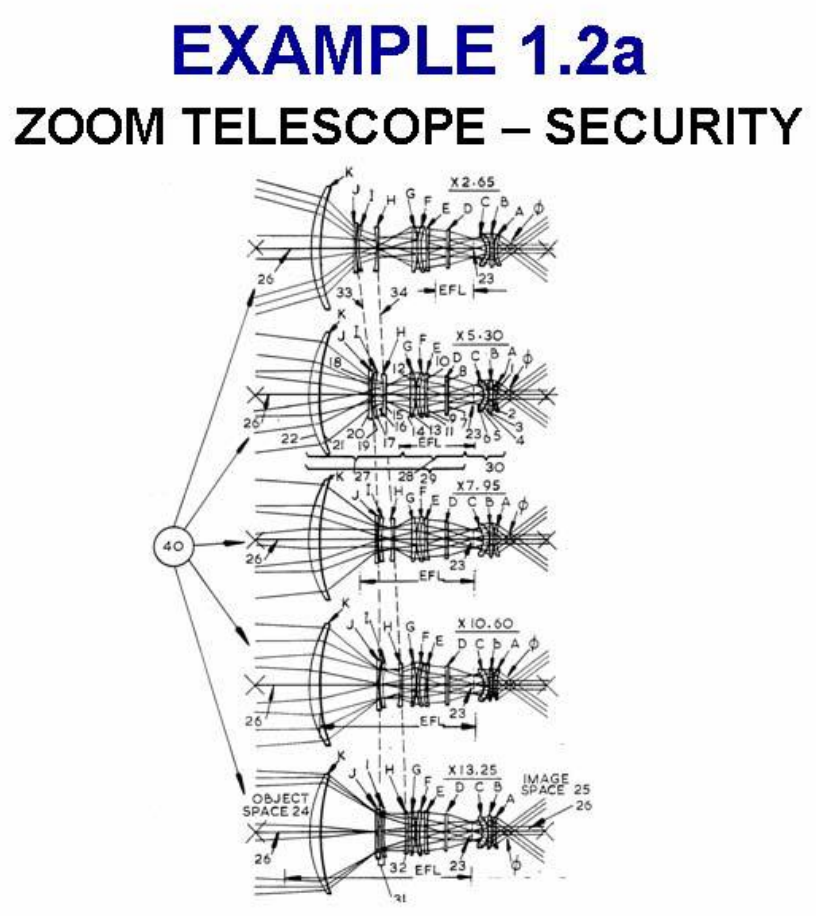

SPIE Europe
Optical Systems Design

"Optical system design reliance on technology development"

\section{ScotOptix}

\section{EXAMPLE 1.2b \\ ZOOM TELESCOPE - SECURITY}

Compact Mechanically Compensated Zoom System

Zoom Ratio $=5 x$ Exit Pupil $\varnothing=10 \mathrm{~mm} \&$ FOV $\varnothing=72^{\circ}$ Waveband $=8-13 \mu \mathrm{m}$

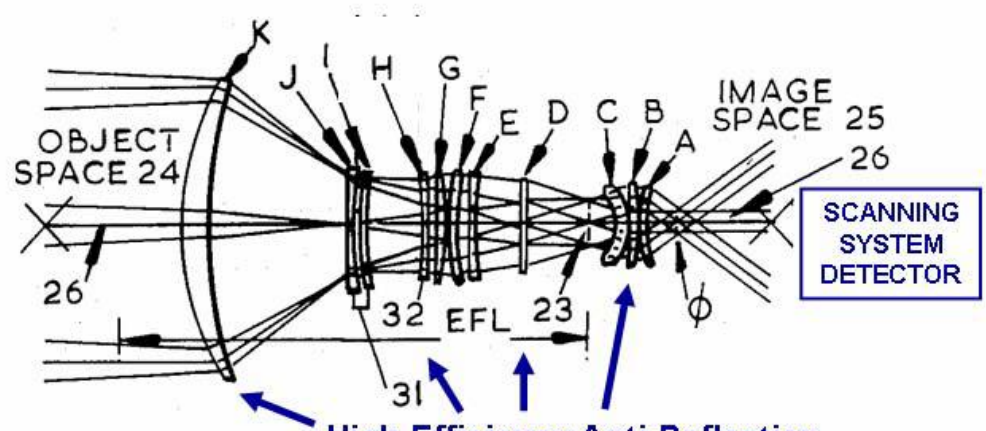

\begin{tabular}{|c|l|}
\hline \multicolumn{2}{|c|}{ KEY } \\
TECHNOLOGY \\
\hline & MATERIAL \\
\hline$\checkmark$ & COATING \\
\hline & SURFACE \\
\hline BENEFITS \\
\hline COMPACT \\
\hline ISSUES \\
\hline NONE \\
\hline
\end{tabular}

High Efficiency Anti-Reflection

Coatings Throughout

US Pat. No. US4,659,171 A1 I.A.Neil Apr. 21, 1987

SPIE Europe
Optical Systems Design

Glasgow, Scotland, United Kingdom - 2nd September 2008

10 


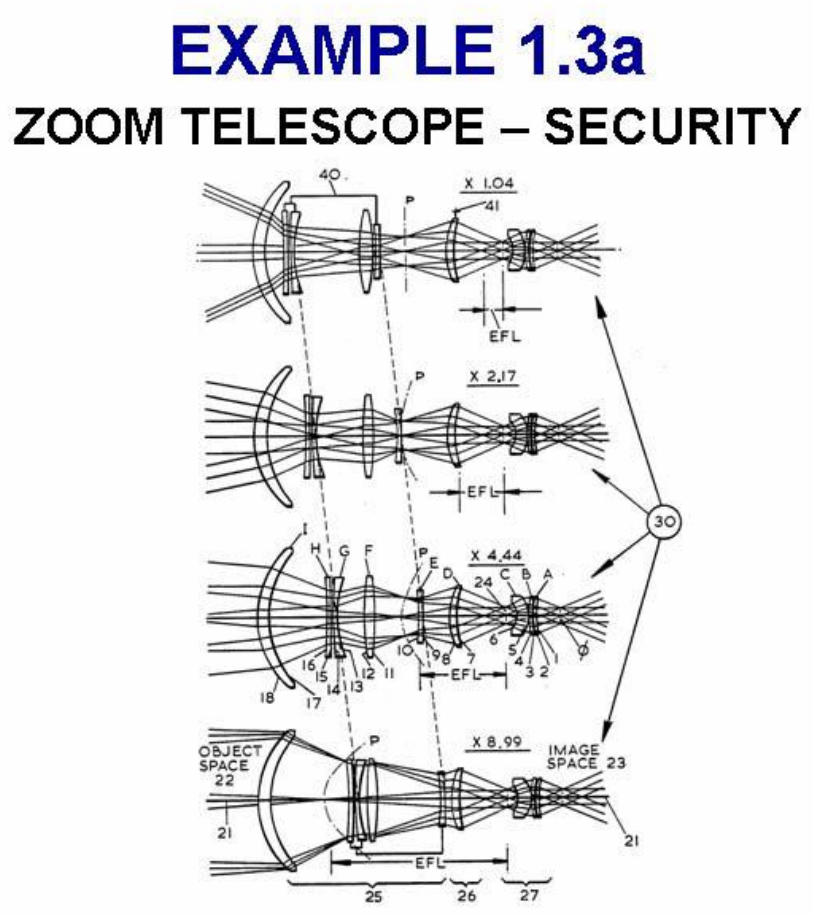

SPIE Europe
Optical Systems Design

"Optical system design reliance on technology development"

\section{ScotOptix}

\section{EXAMPLE 1.3b \\ ZOOM TELESCOPE - SECURITY}

Compact Optically Compensated Zoom System

Zoom Ratio $=9 x$ Exit Pupil $\varnothing=14.4 \mathrm{~mm} \&$ FOV $\varnothing=60^{\circ}$ Waveband $=8-13 \mu \mathrm{m}$

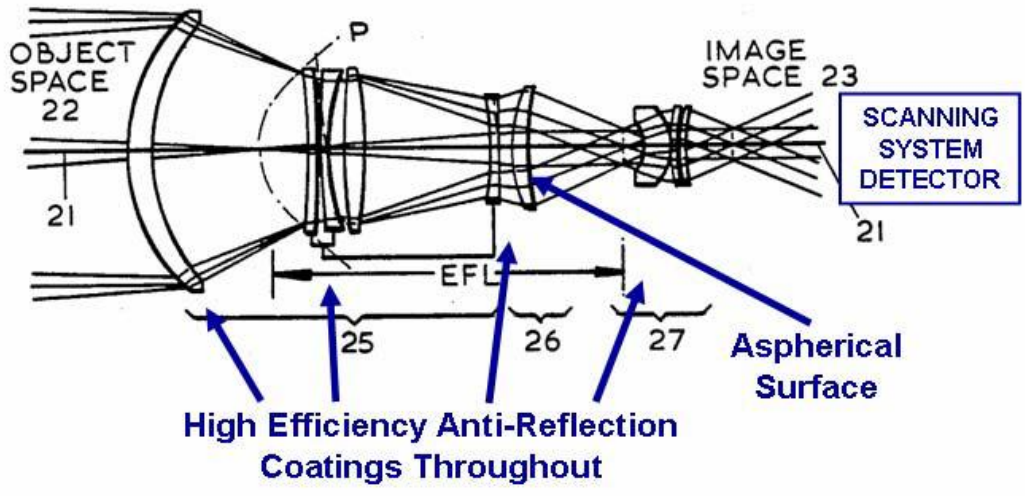

US Pat. No. 4,632,498 A1 I.A.Neil Dec. 30, 1986

\begin{tabular}{|c|l|}
\hline \multicolumn{2}{|c|}{ KEY } \\
TECHNOLOGY \\
\hline & MATERIAL \\
\hline$\checkmark$ & COATING \\
\hline$\checkmark$ & SURFACE \\
\hline \multicolumn{2}{|c|}{ BENEFITS } \\
\hline \multicolumn{2}{|c|}{ COMPACT } \\
\hline SIMPLE \\
MECHANICS \\
\hline \multicolumn{2}{|c|}{ ISSUES } \\
\hline $\begin{array}{c}\text { FOCUS DRIFT } \\
\text { THROUGH ZOOM }\end{array}$ \\
\hline ASPHERE COST \\
\hline
\end{tabular}

SPIE Europe
Optical Systems Design

Glasgow, Scotland, United Kingdom - 2nd September 2008 


\section{EXAMPLE 1.4 OBJECTIVE - SECURITY}

Passively Athermalized \& Color Corrected Air Spaced Doublet with Diffractive Surface

Waveband $=8-13 \mu \mathrm{m}$ (possibly $3-5 \mu \mathrm{m}$ depending on materials)

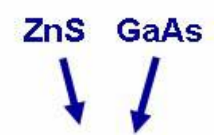

$9 千=$

Diffractive

Surface

US Pat. No. 5,504,628 A1 J.F.Borchard Apr. 2, 1996

\begin{tabular}{|c|l|}
\hline \multicolumn{2}{|c|}{ KEY } \\
TECHNOLOGY \\
\hline$\checkmark$ & MATERIAL \\
\hline & COATING \\
\hline$\checkmark$ & SURFACE \\
\hline BENEFITS \\
\hline SOLID STATE \\
\hline ROBUST \\
\hline \multicolumn{2}{|c|}{ ISSUES } \\
\hline $\begin{array}{c}\text { SECONDARY } \\
\text { COLOR }\end{array}$ \\
\hline LONG LENGTH \\
\hline
\end{tabular}

13

SPIE Europe

Optical Systems Design

Glasgow, Scotland, United Kingdom - 2nd September 2008

\section{ScotOptix}

"Optical system design reliance on technology development"

\section{EXAMPLE 1.5a \\ ZOOM OBJECTIVE - SECURITY}

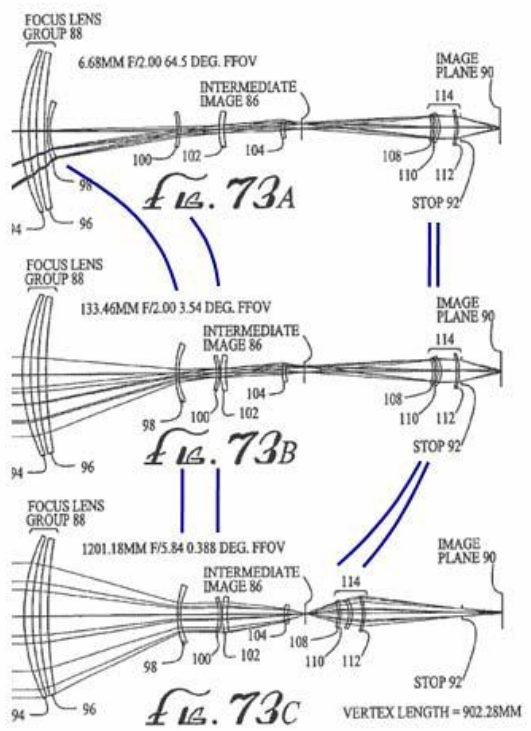

SPIE Europe
Optical Systems Design

Glasgow, Scotland, United Kingdom - 2nd September 2008 


\section{EXAMPLE 1.5b \\ ZOOM OBJECTIVE - SECURITY}

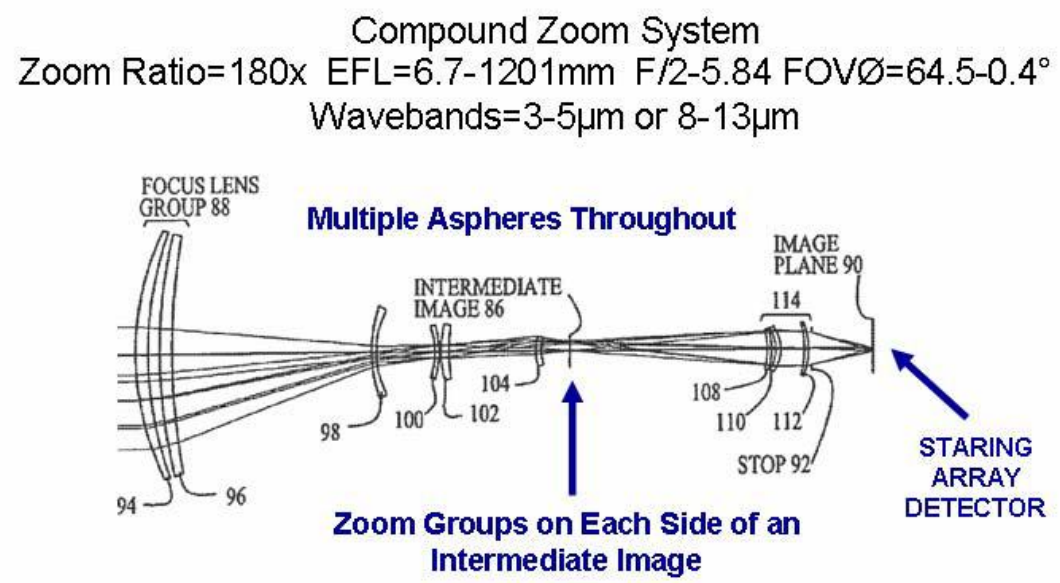

\begin{tabular}{|c|l|}
\hline \multicolumn{2}{|c|}{ KEY } \\
TECHNOLOGY \\
\hline & MATERIAL \\
\hline$\checkmark$ & COATING \\
\hline$\checkmark$ & SURFACE \\
\hline BENEFITS \\
\hline HIGH ZOOM \\
RATIO \\
\hline ISSUES \\
\hline COMPLEX \\
MECHANICS \\
\hline IMAGE F/NO \\
VARIES \\
\hline ASPHERE COST \\
\hline
\end{tabular}

US Pat. No. 7,224,535 B2 I.A.Neil May 29, 2007

SPIE Europe
Optical Systems Design Glasgow, Scotland, United Kingdom - 2nd September 2008

"Optical system design reliance on technology development"

\section{WAVEBAND 2 VISIBLE}




\section{EXAMPLE 2.1 \\ COMPACT CAMERA ZOOM OBJECTIVE - PHOTOGRAPHIC CONSUMER}

Zoom Objective System with $2 x$ Zoom Ratio

$E F L=35.7-68.5 \mathrm{~mm}$ F/3.5-6.8 Image $\varnothing=43.2 \mathrm{~mm}$ Waveband=Visible

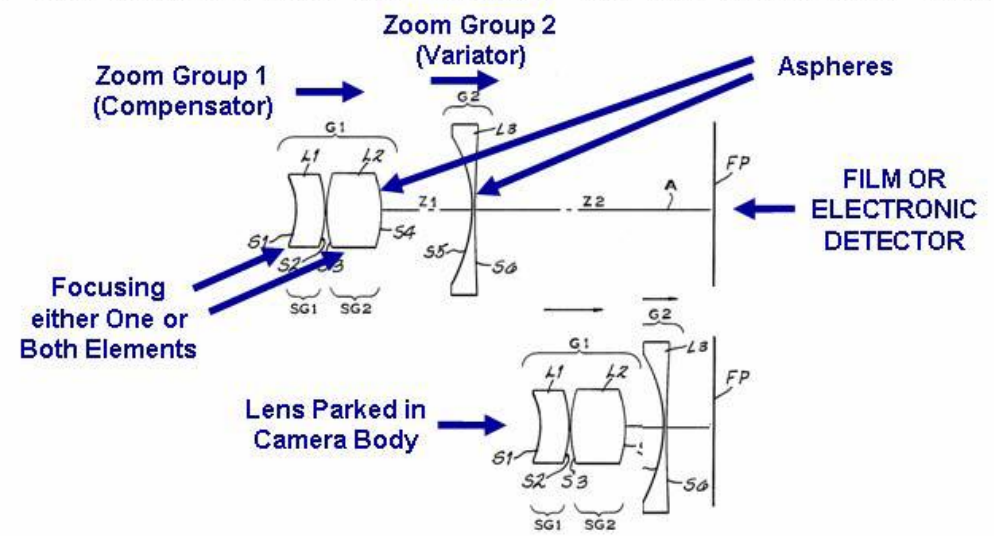

\begin{tabular}{|c|l|}
\hline \multicolumn{2}{|c|}{ KEY } \\
TECHNOLOGY \\
\hline & MATERIAL \\
\hline & COATING \\
\hline$\checkmark$ & SURFACE \\
\hline BENEFITS \\
\hline SIMPLE \\
\hline COMPACT \\
\hline LOW COST \\
\hline ISSUES \\
\hline MOLDED \\
ASPHERES \\
\hline
\end{tabular}

US Pat. No. 4,936,661 A1 E.I.Betensky, M.H.Kreitzer \& J.Moskovich Jun. 26, 1990

SPIE Europe

Optical Systems Design

Glasgow, Scotland, United Kingdom - 2nd September 2008

"Optical system design reliance on technology development"

\section{ScotOptix}

\section{EXAMPLE 2.2a}

TELEPHOTO OBJECTIVE - PHOTOGRAPHIC CINE

Passively Athermalized \& Color Corrected System with Liquid Elements

$E F L=693 \mathrm{~mm} F / 2.75 \mathrm{Image} \varnothing=28.9 \mathrm{~mm}$ Waveband $=435-656 \mathrm{~nm}$

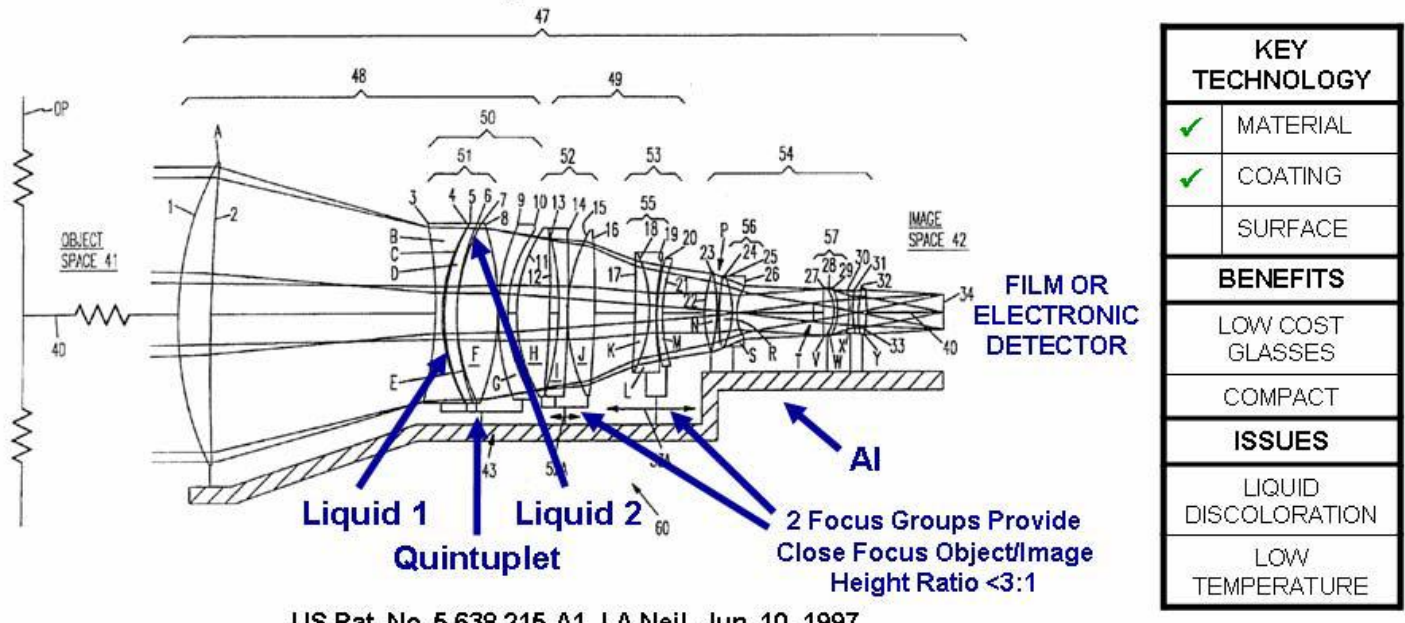

SPIE Europe
Optical Systems Design Glasgow, Scotland, United Kingdom - 2nd September 2008 


\section{EXAMPLE 2.2b \\ TELEPHOTO OBJECTIVE - PHOTOGRAPHIC CINE \\ Quintuplet}

Liquid 1

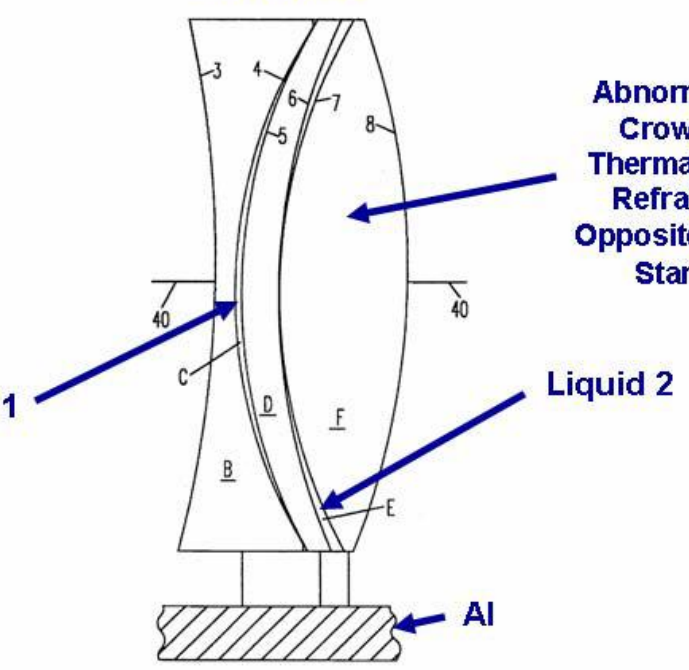

SPIE Europe

Optical Systems Design

"Optical system design reliance on technology development"

\section{EXAMPLE 2.3a \\ MACRO FOCUS ZOOM OBJECTIVE \\ - PHOTOGRAPHIC CINE}

THROUGH ZOOM

(INFINITY FOCUS)

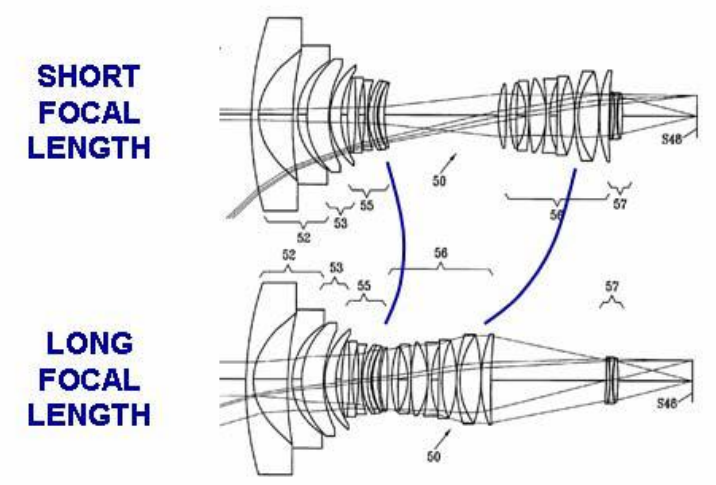




\section{EXAMPLE 2.3b \\ MACRO FOCUS ZOOM OBJECTIVE \\ - PHOTOGRAPHIC CINE}

THROUGH FOCUS

(SHORT FOCAL LENGTH)

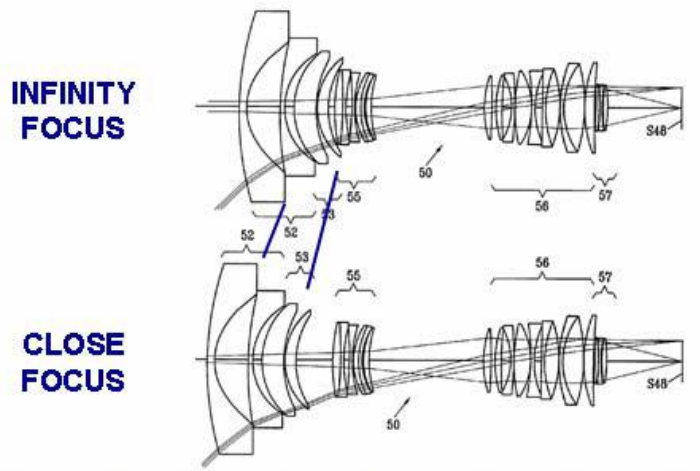

Close Focus Object/Image Height Ratio $=2.5: 1$ (At Long Focal Length)

SPIE Europe

Optical Systems Design

Glasgow, Scotland, United Kingdom - 2nd September 2008

"Optical system design reliance on technology development"

\section{ScotOptix}

\section{EXAMPLE 2.3C \\ ZOOM OBJECTIVE - PHOTOGRAPHIC CINE}

Macro Focus Zoom System with $3.5 \times$ Zoom Ratio

$E F L=14.5-50 \mathrm{~mm} F / 2.2$ Image $\varnothing=28.9 \mathrm{~mm}$ Waveband $=455-644 \mathrm{~nm}$

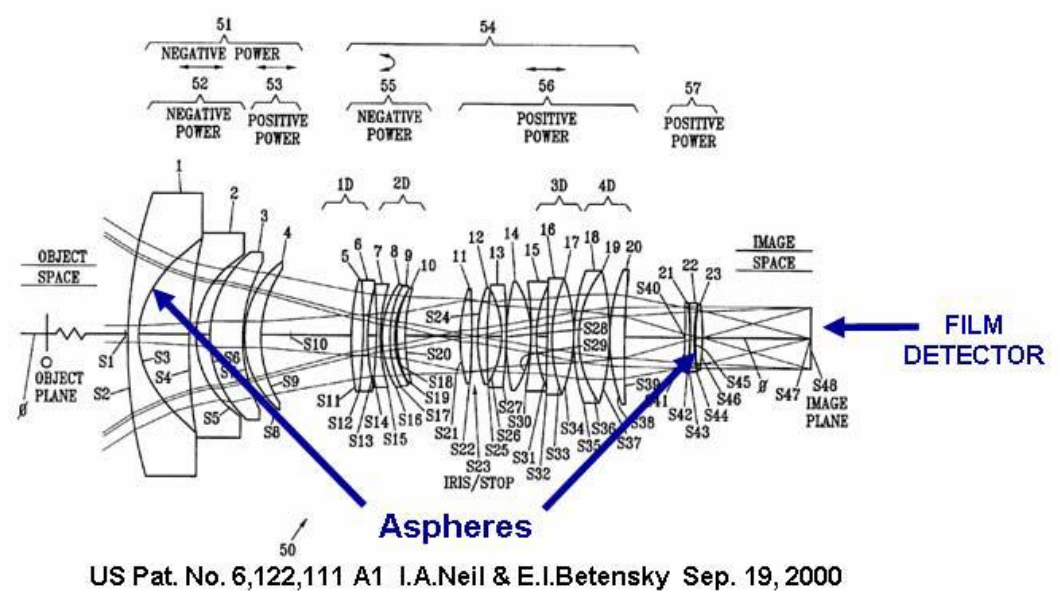

\begin{tabular}{|c|l|}
\hline \multicolumn{2}{|c|}{ KEY } \\
TECHNOLOGY \\
\hline & MATERIAL \\
\hline$\checkmark$ & COATING \\
\hline$\checkmark$ & SURFACE \\
\hline BENEFITS \\
\hline VERSATILE \\
\hline $\begin{array}{c}\text { FIXED FOCAL } \\
\text { LENGTH OPTION }\end{array}$ \\
\hline ISSUES \\
\hline COMPLEX \\
MECHANICS \\
\hline ASPHERE \\
COST \\
\hline
\end{tabular}

SPIE Europe
Optical Systems Design

Glasgow, Scotland, United Kingdom - 2nd September 2008 


\section{EXAMPLE 2.4a \\ ZOOM OBJECTIVE - PHOTOGRAPHIC CINE}

THROUGH ZOOM

(INFINITY FOCUS)

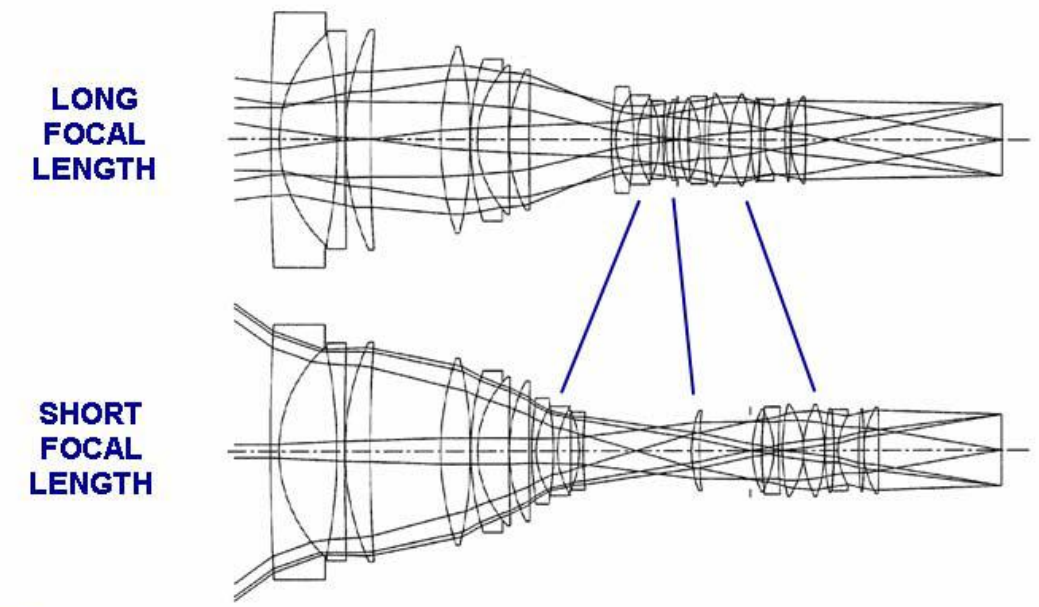

SPIE Europe

Optical Systems Design

Glasgow, Scotland, United Kingdom - 2nd September 2008

"Optical system design reliance on technology development"

\section{ScotOptix}

\section{EXAMPLE 2.4b \\ ZOOM OBJECTIVE - PHOTOGRAPHIC CINE}

Compact Zoom Objective System with 4.7x Zoom Ratio

$\mathrm{EFL}=19-90 \mathrm{~mm} F / 2.7$ Image $\varnothing=27.8 \mathrm{~mm}$ Waveband $=455-644 \mathrm{~nm}$

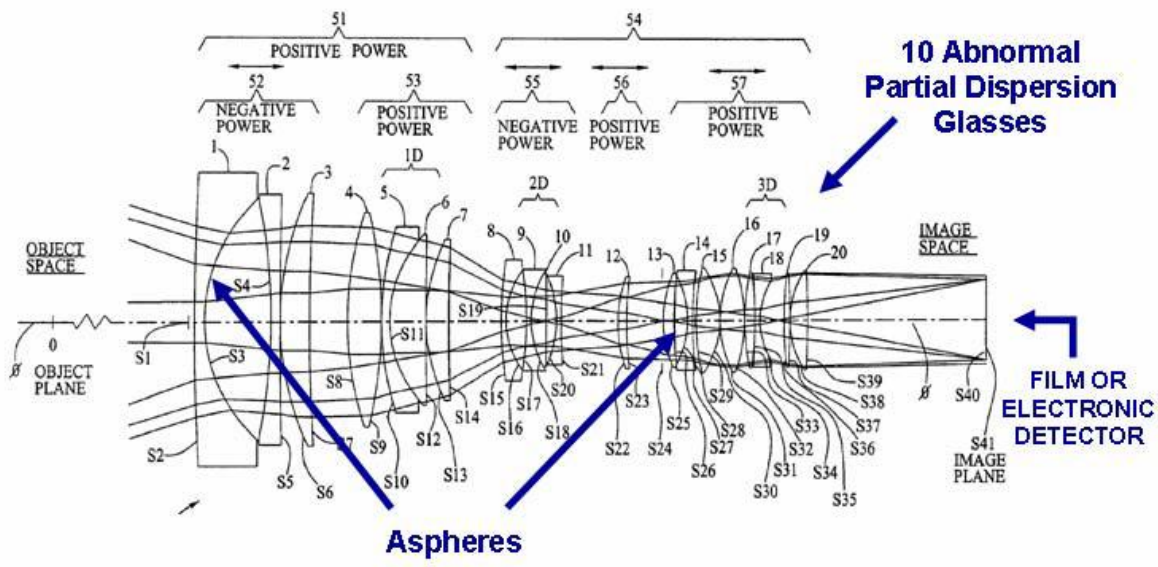

US Pat. No. 7,123,421 B1 J.Moskovich, I.A.Neil \& T.Yamanashi Oct. 17, 2006

\begin{tabular}{|c|l|}
\hline \multicolumn{2}{|c|}{ KEY } \\
TECHNOLOGY \\
\hline$\checkmark$ & MATERIAL \\
\hline$\checkmark$ & COATING \\
\hline$\checkmark$ & SURFACE \\
\hline BENEFITS \\
\hline COMPACT \\
\hline VERSATILE \\
\hline $\begin{array}{c}\text { FIXED FOCAL } \\
\text { LENGTH OPTION }\end{array}$ \\
\hline \multicolumn{2}{|c|}{ ISSUES } \\
\hline COMPLEX \\
MECHANICS \\
\hline ASPHERE \\
COST \\
\hline
\end{tabular}

SPIE Europe

Optical Systems Design

Glasgow, Scotland, United Kingdom - 2nd September 2008 


\section{EXAMPLE 2.5}

\section{OBJECTIVE - PHOTOGRAPHIC PROSUMER}

Telephoto System with Diffractive Surface

$\mathrm{EFL}=780 \mathrm{~mm} F / 5.8 \mathrm{Image} \varnothing=43.2 \mathrm{~mm}$ Waveband $=435-656 \mathrm{~nm}$

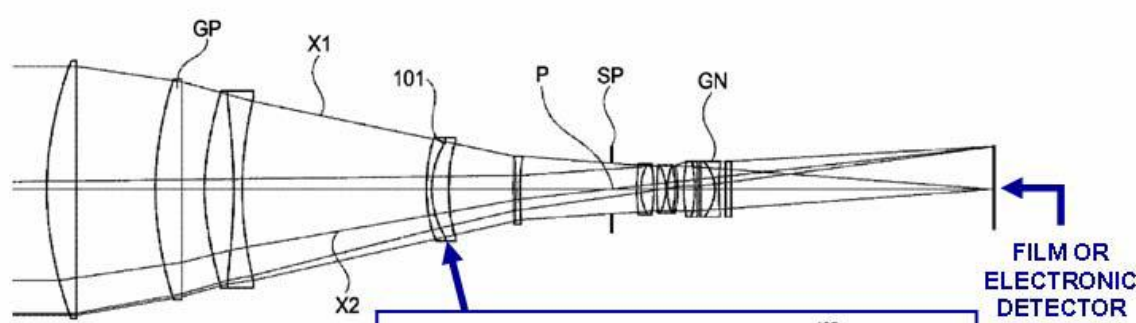

\begin{tabular}{|c|l|}
\hline \multicolumn{2}{|c|}{ KEY } \\
TECHNOLOGY \\
\hline$\checkmark$ & MATERIAL \\
\hline & COATING \\
\hline$\checkmark$ & SURFACE \\
\hline BENEFITS \\
\hline COMPACT \\
\hline COLOR \\
CORRECTED \\
\hline REDUCED NO. OF \\
EXOTIC GLASSES \\
\hline ISSUES \\
\hline FLARE \\
\hline
\end{tabular}

US Pat. Pub. No. 200810088950 A1 H.Endo Apr. 17, 2008

SPIE Europe

Optical Systems Design

Glasgow, Scotland, United Kingdom - 2nd September 2008

"Optical system design reliance on technology development"

\section{ScotOptix}

\section{EXAMPLE 2.6a \\ ZOOM OBJECTIVE - PHOTOGRAPHIC HDTV}

THROUGH ZOOM

(INFINITY FOCUS)

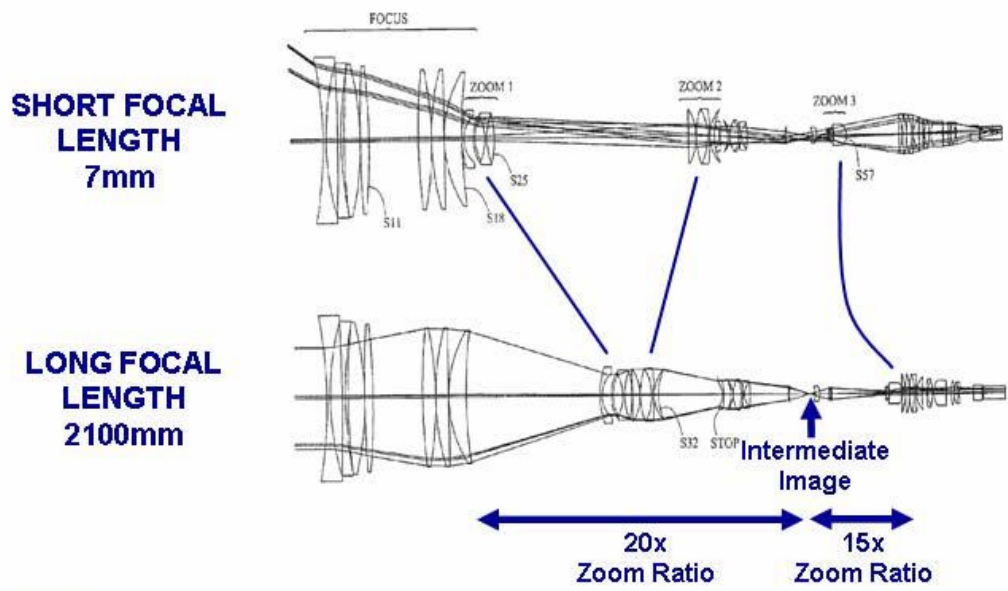

SPIE Europe

Optical Systems Design 


\section{EXAMPLE 2.6b \\ ZOOM OBJECTIVE - PHOTOGRAPHIC HDTV}

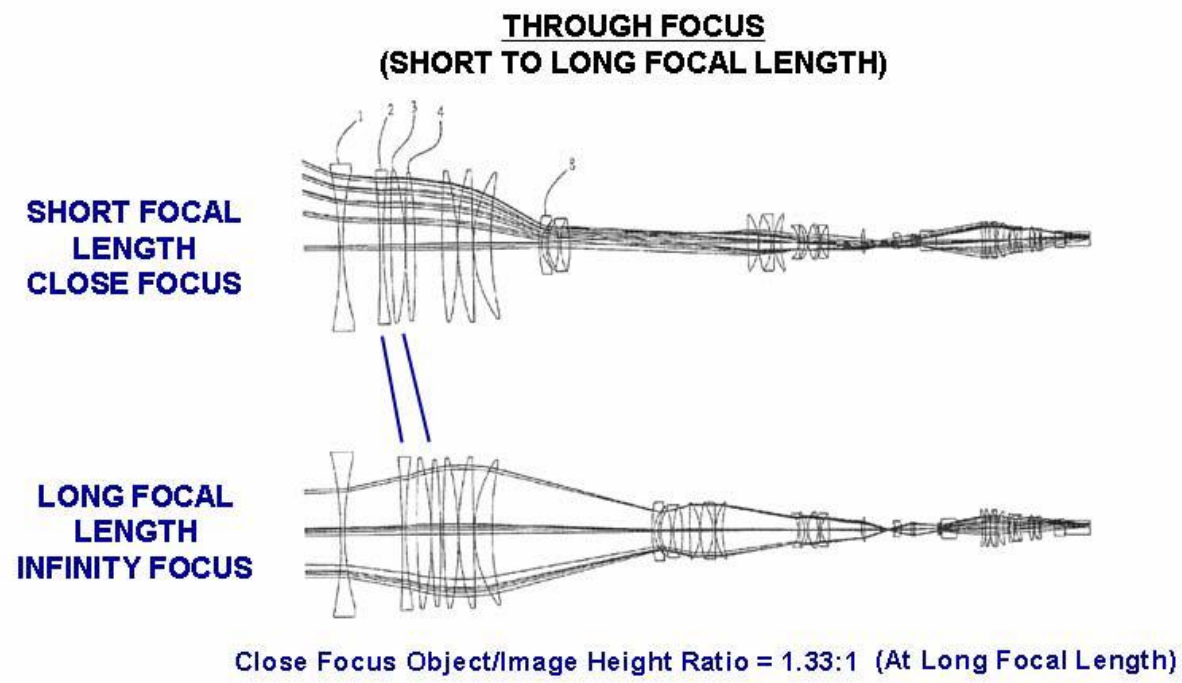

SPIE Europe

Optical Systems Design

Glasgow, Scotland, United Kingdom - 2nd September 2008

"Optical system design reliance on technology development"

\section{ScotOptix}

\section{EXAMPLE 2.6C \\ ZOOM OBJECTIVE - PHOTOGRAPHIC HDTV}

Compound Zoom System with $300 \times$ Zoom Ratio

$E F L=7-2100 \mathrm{~mm} \quad F / 2-13$ Image $\varnothing=11 \mathrm{~mm}$ Waveband=Visible

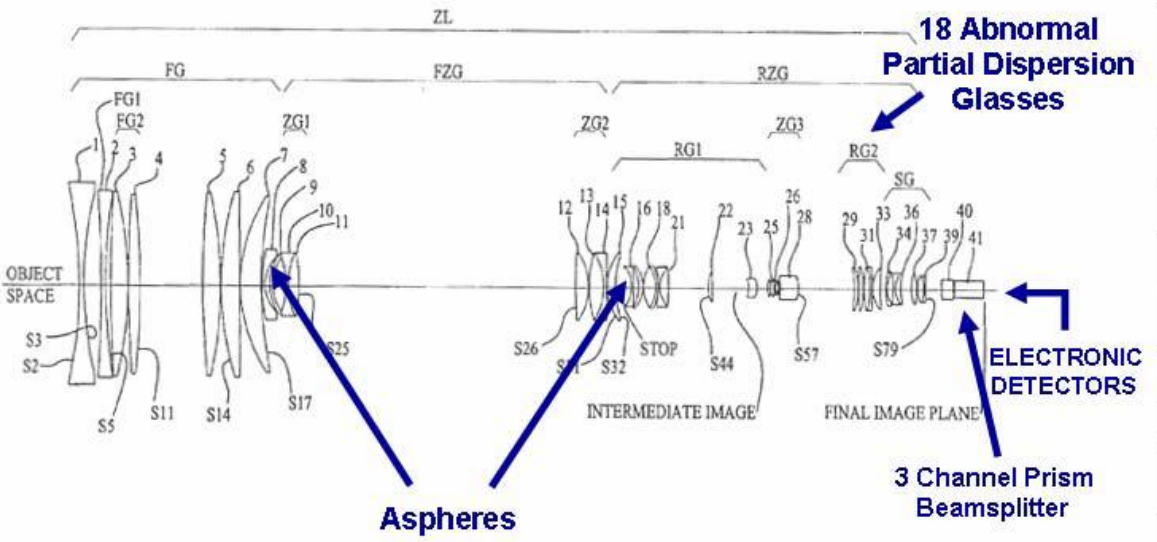

US Pat. No. 6,691,188 B2 E.I.Betensky, J.B.Caldwell, I.A.Neil \& T.Yamanashi Nov. 1, 2005

\begin{tabular}{|c|l|}
\hline \multicolumn{2}{|c|}{ KEY } \\
\multicolumn{2}{|c|}{ TECHNOLOGY } \\
\hline$\checkmark$ & MATERIAL \\
\hline$\checkmark$ & COATING \\
\hline$\checkmark$ & SURFACE \\
\hline BENEFITS \\
\hline $\begin{array}{c}\text { LARGE ZOOM } \\
\text { RATIO }\end{array}$ \\
\hline \multicolumn{2}{|c|}{ VERSATILE } \\
\hline \multicolumn{2}{|c|}{ ISSUES } \\
\hline \multicolumn{2}{|c|}{ COMPLEX } \\
MECHANICS \\
\hline ASPHERE COST \\
\hline
\end{tabular}

28

xxiv 


\section{WAVEBAND 3 ULTRAVIOLET}

SPIE Europe

\section{ScotOptix}

\section{EXAMPLE 3.1 \\ PROJECTION RELAY LENS - MICROLITHOGRAPHIC}

All Refractive Projection System

RELAY=5:1 NA=0.57 Image $\varnothing=31.2 \mathrm{~mm}$ Wavelengths $=193,248 \& 365 \mathrm{~nm}$

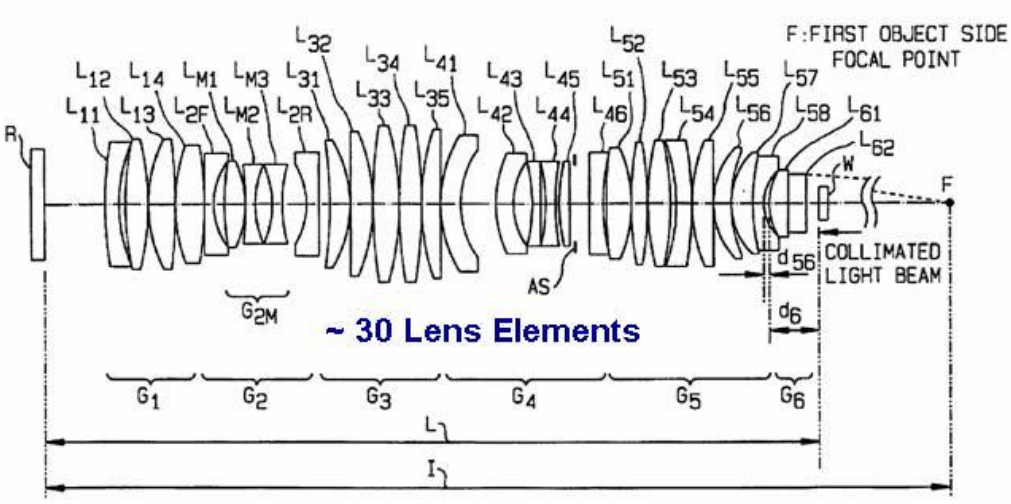

\begin{tabular}{|c|l|}
\hline \multicolumn{2}{|c|}{ KEY } \\
TECHNOLOGY \\
\hline & MATERIAL \\
\hline$\checkmark$ & COATING \\
\hline$\checkmark$ & SURFACE \\
\hline \multicolumn{2}{|c|}{ BENEFITS } \\
\hline RESOLUTION \\
\hline \multicolumn{2}{|c|}{ ISSUES } \\
\hline $\begin{array}{c}\text { INHOMOGENEITY \& } \\
\text { BIREFRINGENCE }\end{array}$ \\
\hline SURFACE QUALITY \\
\hline \multicolumn{2}{|c|}{ ALIGNMENT } \\
\hline
\end{tabular}

US Reissued Pat. No. RE 37,846E H.Matsuzawa, M.Kobayashi, K.Endo \& Y.Suenaga Sep. 17, 2002

SPIE Europe

Optical Systems Design 


\section{EXAMPLE 3.2 \\ PROJECTION RELAY LENS - MICROLITHOGRAPHIC}

Refractive/Reflective Projection System

RELAY $=4: 1 \quad N A=0.45$ Image $\varnothing=30 \mathrm{~mm}$ Wavelengths $=240-256 \mathrm{~nm}$

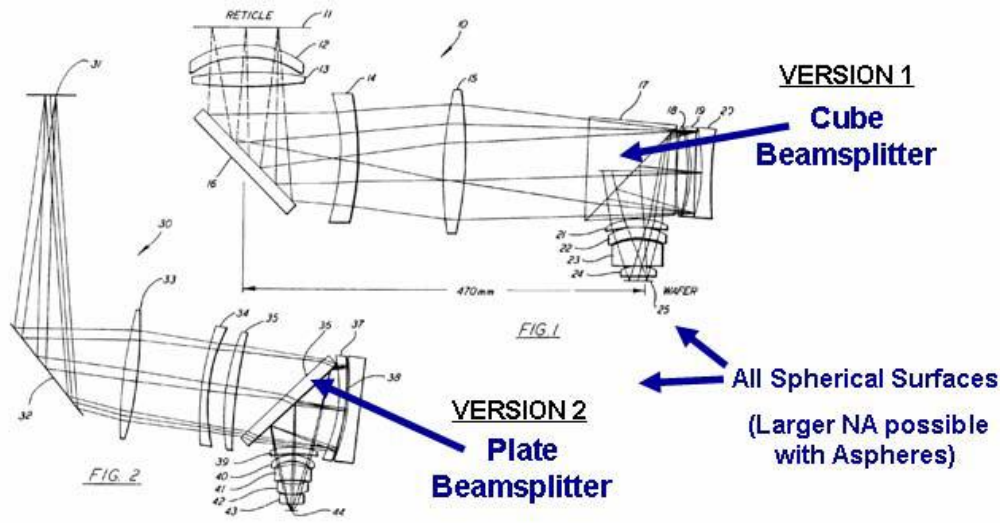

\begin{tabular}{|c|l|}
\hline \multicolumn{2}{|c|}{ KEY } \\
TECHNOLOGY \\
\hline$\checkmark$ & MATERIAL \\
\hline$\checkmark$ & COATING \\
\hline & SURFACE \\
\hline \multicolumn{2}{|c|}{ BENEFITS } \\
\hline \multicolumn{2}{|c|}{ RESOLUTION } \\
\hline \multicolumn{2}{|c|}{ ISSUES } \\
\hline \multicolumn{2}{|c|}{ ALIGNMENT } \\
\hline
\end{tabular}

US Pat. No. 4,953,960 A1 D.M.Williamson Sep. 4, 1990

SPIE Europe

Optical Systems Design

Glasgow, Scotland, United Kingdom - 2nd September 2008

31

"Optical system design reliance on technology development"

ScotOptix

\section{EXAMPLE 3.3}

\section{PROJECTION RELAY OPTICS - MICROLITHOGRAPHIC}

All Reflective Projection System

RELAY $=4: 1 \quad N A=0.25$ Image $\varnothing=31 \mathrm{~mm}$ Wavelengths $=13.4 \mathrm{~nm} \&<200 \mathrm{~nm}$

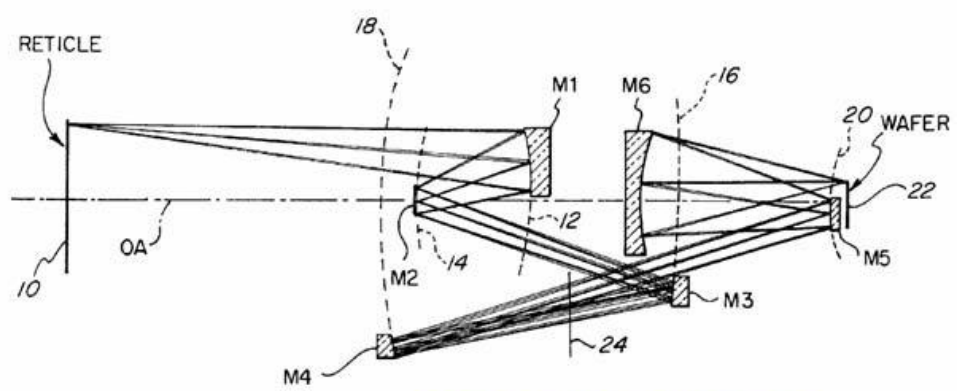

6 Mirrors with 6 Aspheres

US Pat. No. 5,815,310 A1 D.M.Williamson Sep. 29, 1998

\begin{tabular}{|c|l|}
\hline \multicolumn{2}{|c|}{$\begin{array}{c}\text { KEY } \\
\text { TECHNOLOGY }\end{array}$} \\
\hline & MATERIAL \\
\hline$\checkmark$ & COATING \\
\hline$\checkmark$ & SURFACE \\
\hline BENEFITS \\
\hline HIGH \\
RESOLUTION \\
\hline \multicolumn{2}{|c|}{ ISSUES } \\
\hline @13.4nM <10\% \\
TRANSMISSION \\
WITH COATINGS \\
\hline ASPHERE COST \\
\hline ALIGNMENT \\
\hline
\end{tabular}




\section{WAVEBAND 4 MULTIPLE}

SPIE Europe

\section{EXAMPLE 4.1 OBJECTIVE - SECURITY}

Dual Waveband System $F / 4.5$ (elev), $F / 1.5$ (azim) \& F/2.3(average) $F O V \varnothing=40^{\circ}$ (elev.) \& 53(azim.)

Wavebands=Visible \& $8-13 \mu \mathrm{m}$

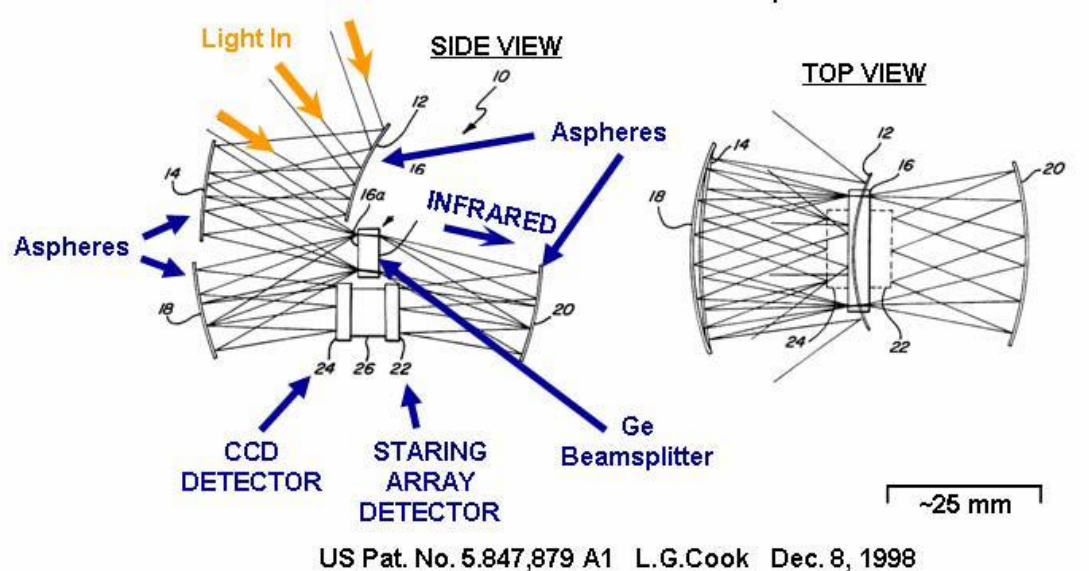

\begin{tabular}{|c|l|}
\hline \multicolumn{2}{|c|}{ KEY } \\
TECHNOLOGY \\
\hline & MATERIAL \\
\hline$\checkmark$ & COATING \\
\hline$\checkmark$ & SURFACE \\
\hline BENEFITS \\
\hline COMPACT \\
\hline SOLID STATE \\
\hline ROBUST \\
\hline ISSUES \\
\hline $\begin{array}{c}\text { ASPHERE } \\
\text { COST }\end{array}$ \\
\hline
\end{tabular}

SPIE Europe

Optical Systems Design 
"Optical system design reliance on technology development"

\section{ScotOptix}

\section{EXAMPLE 4.2a \\ OBJECTIVE - SURVEILLANCE}

Compact Multi-waveband Wide Angle Objective

FOV $15^{\circ}-80^{\circ} \times 360^{\circ}$

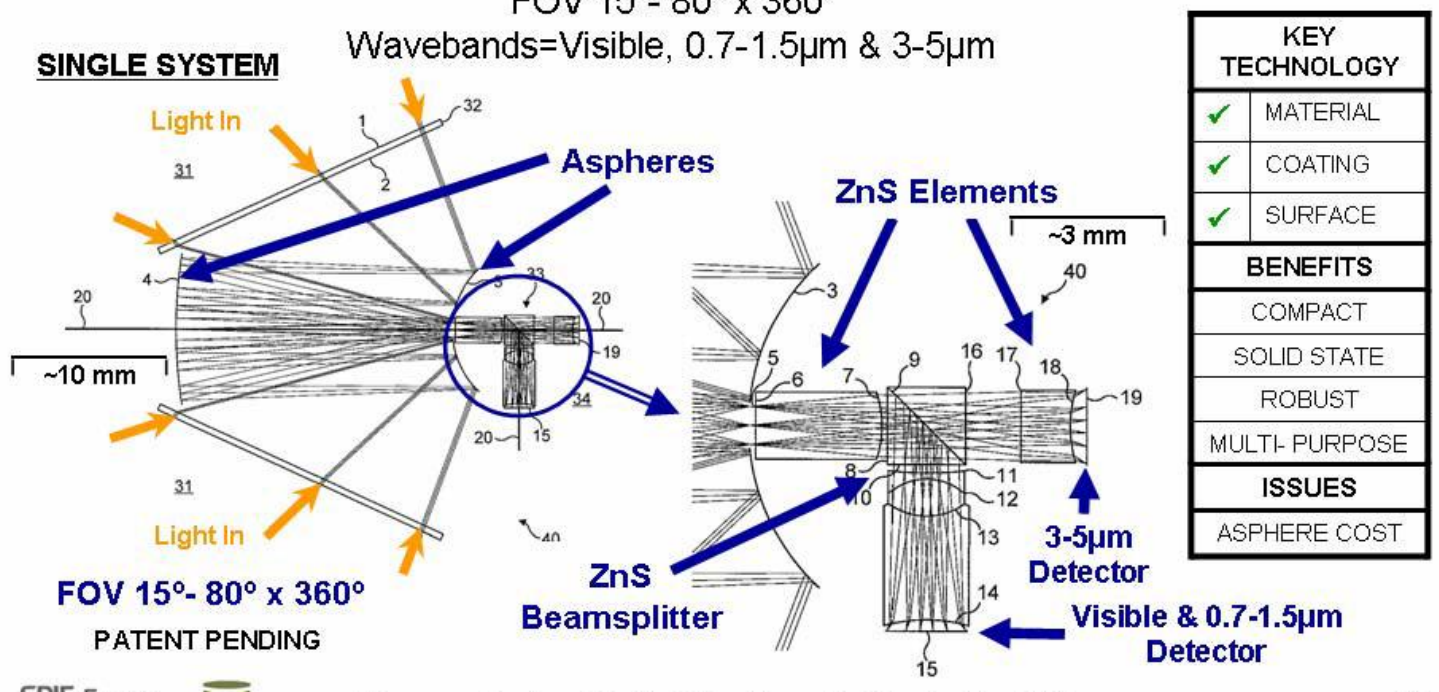

SPIE Europe

Optical Systems Design

"Optical system design reliance on technology development"

\section{ScotOptix}

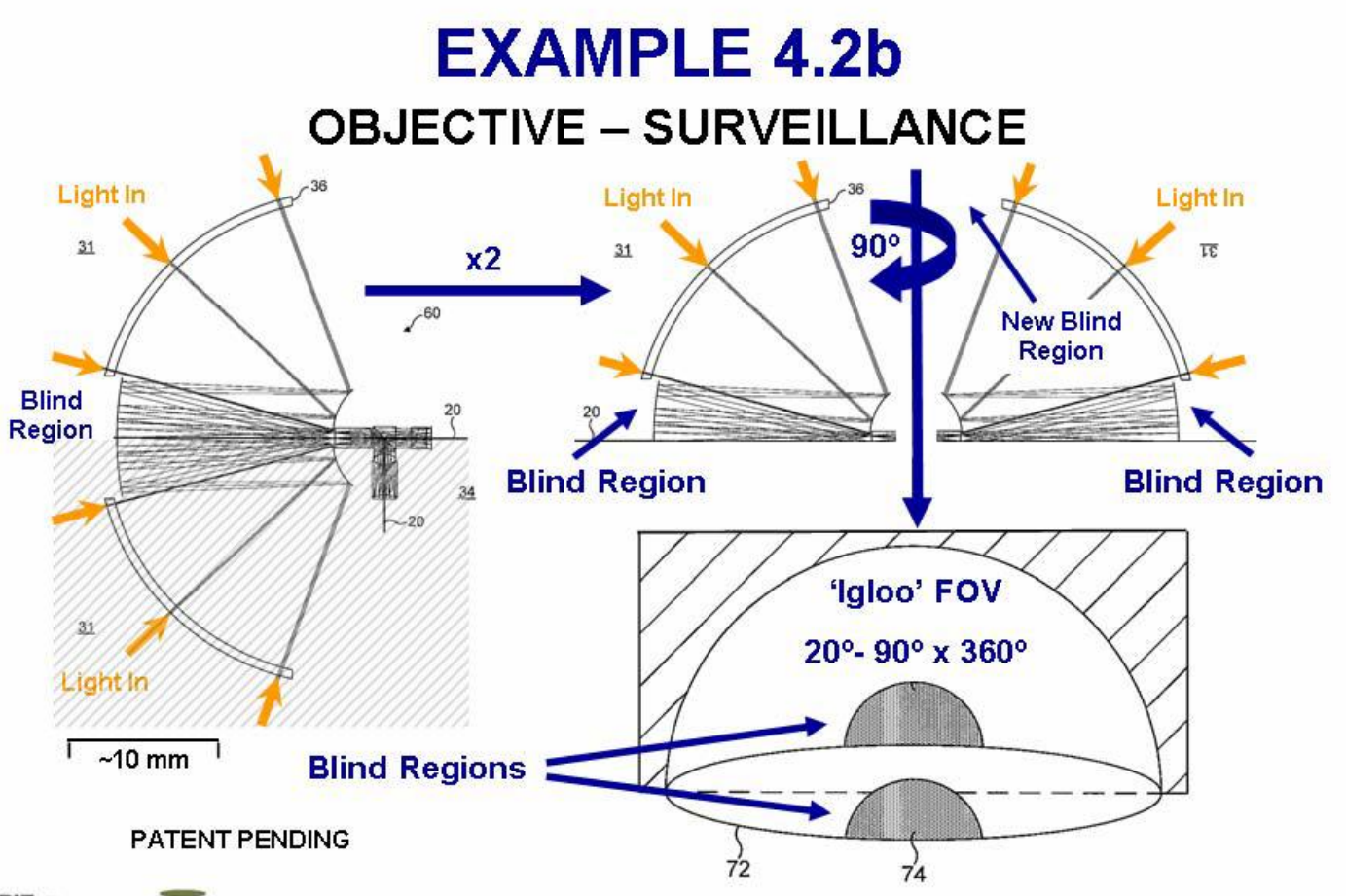

SPIE Europe Glasgow, Scotland, United Kingdom - 2nd September 2008 
"Optical system design reliance on technology development" ScotOptix

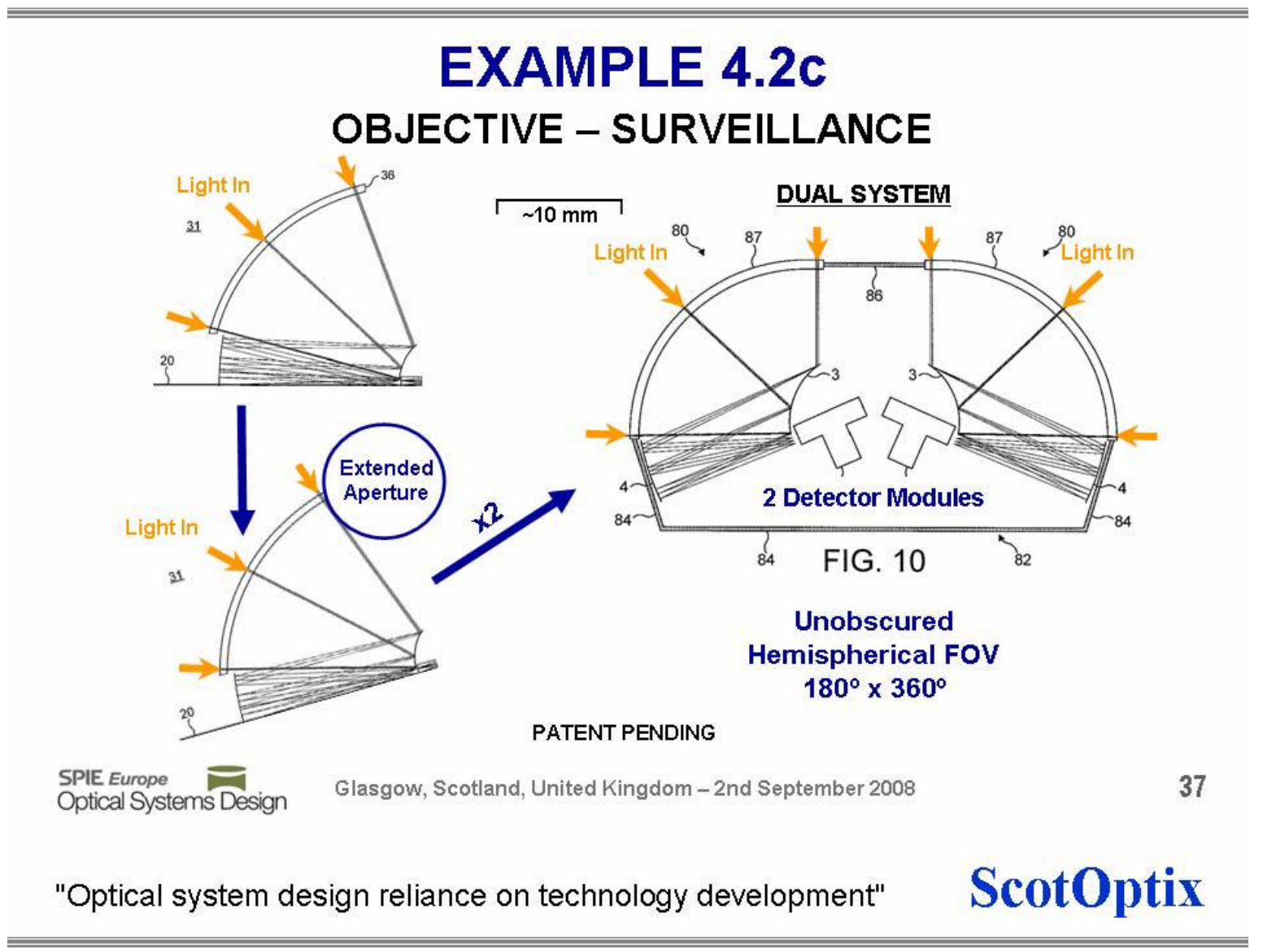

\section{EXAMPLE 4.2d OBJECTIVE - SURVEILLANCE}

SPIE Europe

Optical Systems Design

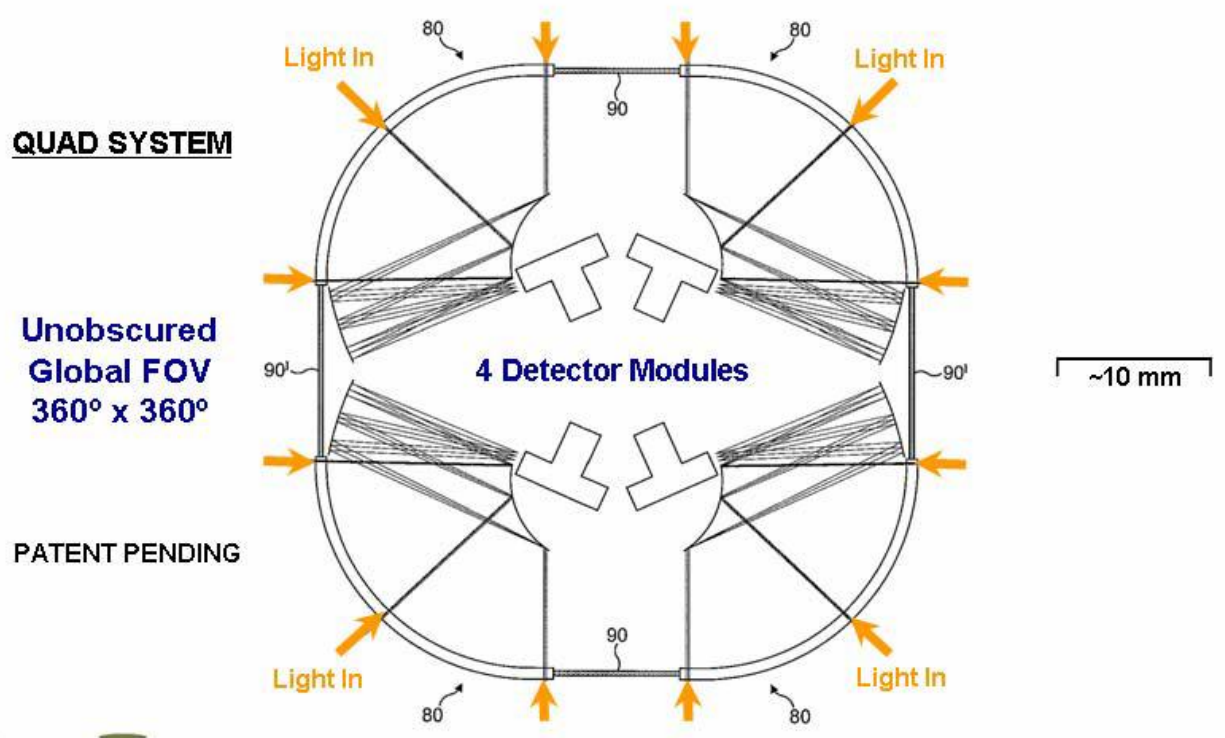

Glasgow, Scotland, United Kingdom - 2nd September 2008 


\section{KEY TECHNOLOGY SUMMARY}

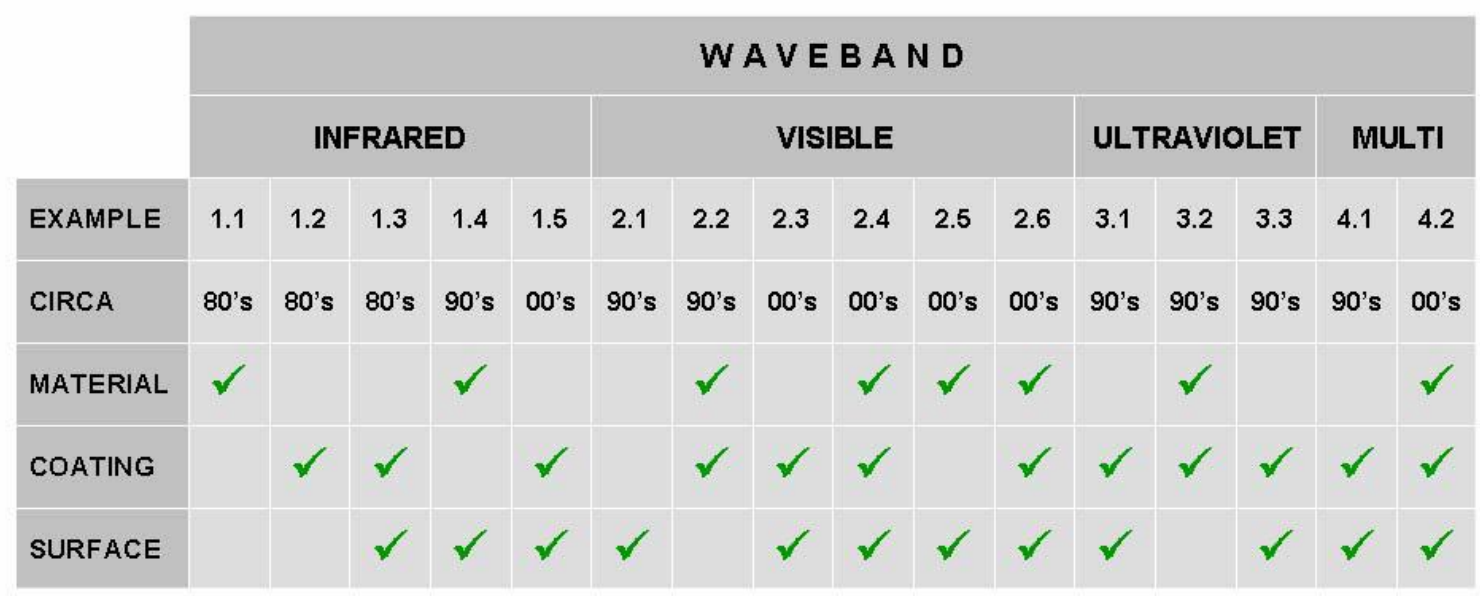

SPIE Europe

Optical Systems Design

\section{CONCLUSION}

- Usually technology provides 'improvements' but occasionally it is 'disruptive' in that it dramatically changes the optical system design such as enabling a new form of design

- In the specific case of disruptive technology this usually appears to happen separately in either materials, coatings or surfaces

- No apparent trend in technology development except:

"Necessity is the mother of invention"

Plato c. $400 \mathrm{BC}$ 


\title{
ACKNOWLEGEMENTS
}

\section{Thanks goes to the following individuals for contributions to this presentation}

\author{
David W. Samuelson \\ David M. Williamson \\ Andy Wood
}

SPIE Europe

Optical Systems Design

Glasgow, Scotland, United Kingdom - 2nd September 2008

41 
Downloaded From: https://www.spiedigitallibrary.org/conference-proceedings-of-spie on 26 Apr 2023

Terms of Use: https://www.spiedigitallibrary.org/terms-of-use 


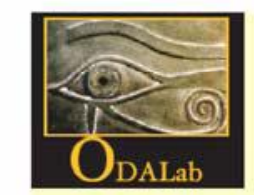

\section{A Perspective on the Design of Head-Worn Displays}

Jannick Rolland with

Ozan Cakmakci, Florian Fournier, and Sophie Vo

CREOL, The College of Optics and Photonics the University of Central Florida

http://odalab.ucf.edu

jannick@odalab.ucf.edu

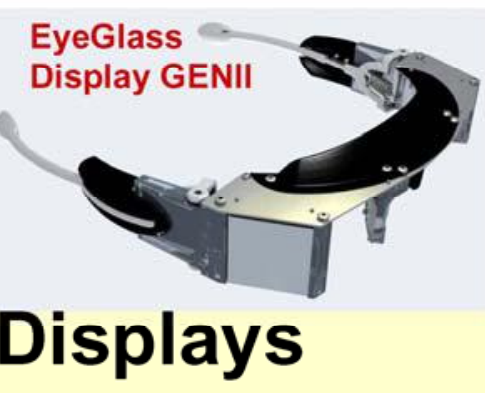




\section{Why Head-Worn Displays?}

Assuming HWDs can be designed aesthetically (which is not a given) to meet with social acceptance:

- Mobility

- Privacy

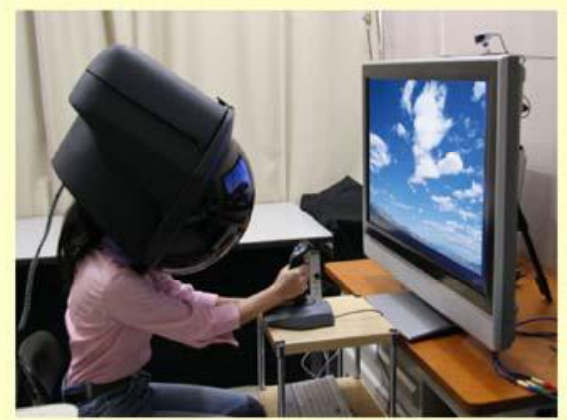

- Constancy: Provides the basis for novel user interfaces that are available constantly (on a demand basis) to the user

\section{Science Fiction Sets Expectations of Where we Aim to Be Going!}

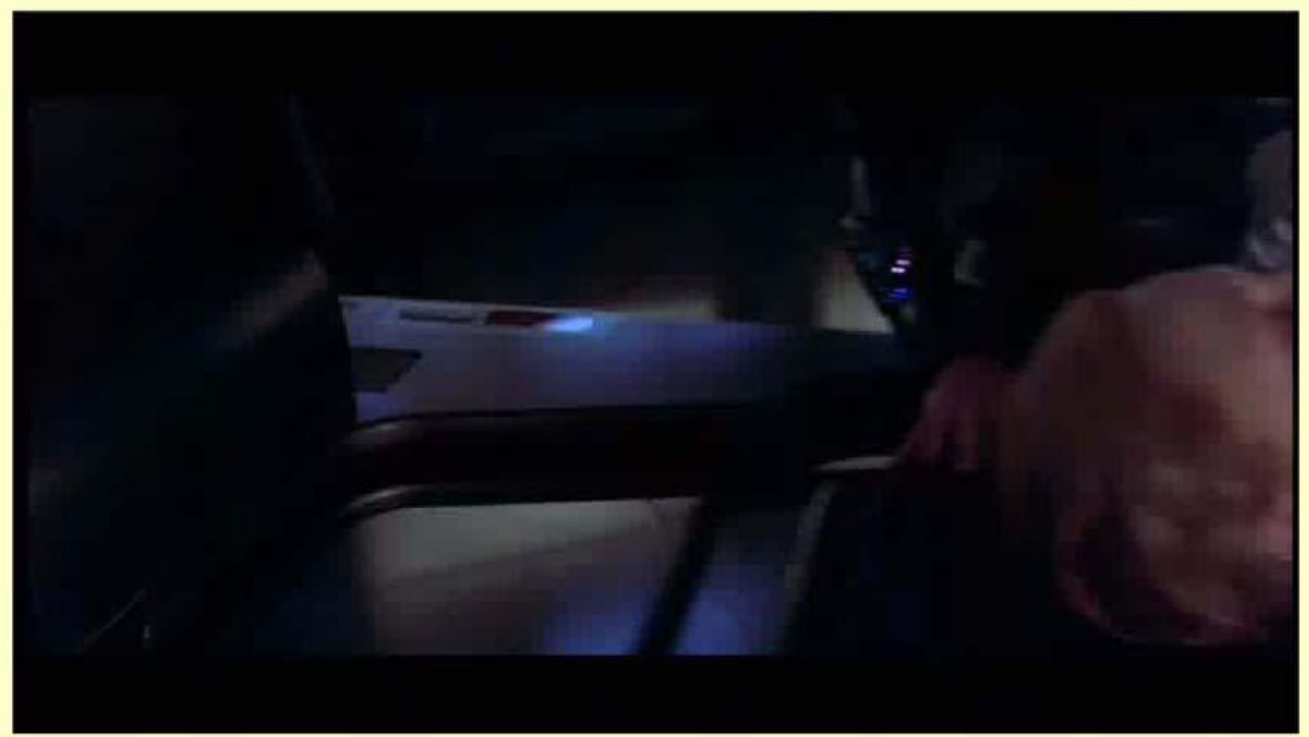

* Goldsman, A. (1998). Lost in Space. New Line Cinema 


\section{Medical Rooms of the Future}

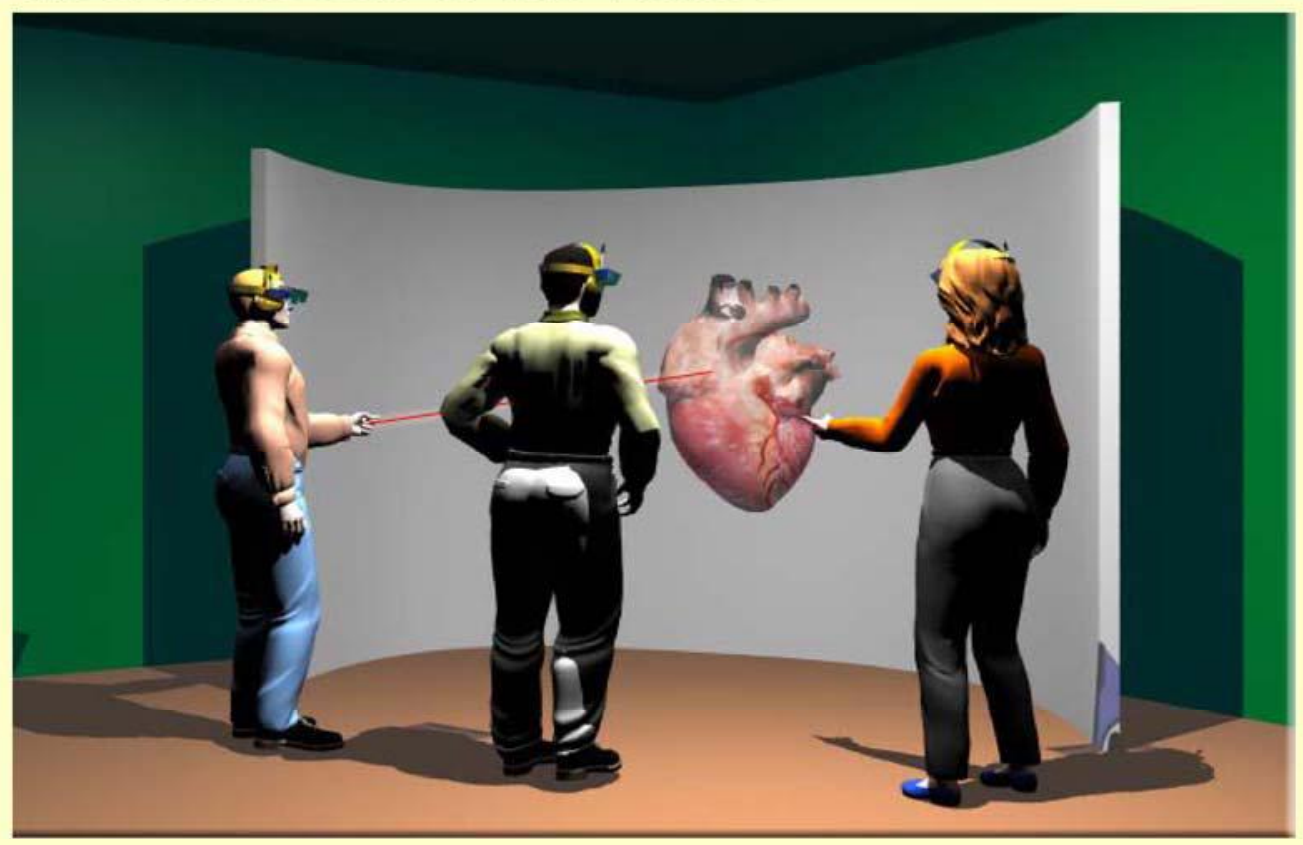

\section{Telemedicine: Face to Face Teleportal}

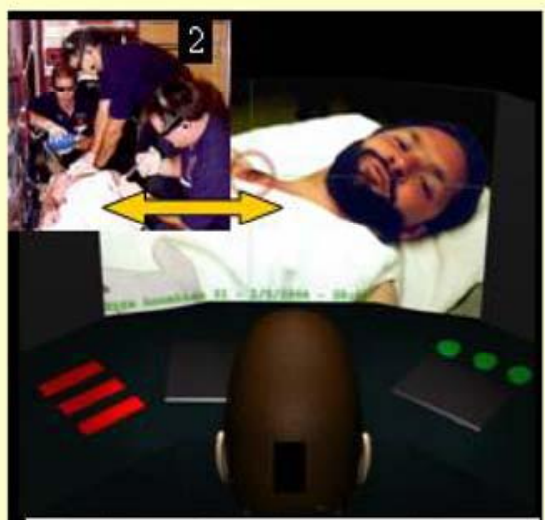

Fig 1. Vision of "see-thun-my-eyes"

capability. (1) Doctor in local control room

guides (2) remote treatment via stereoscopic

see-thru headset wom by emergency

technician.

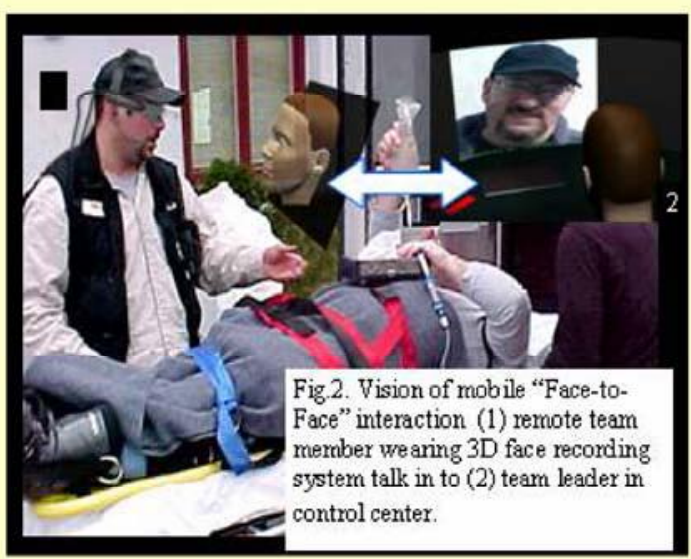

Courtesy of Frank Biocca, MSU 


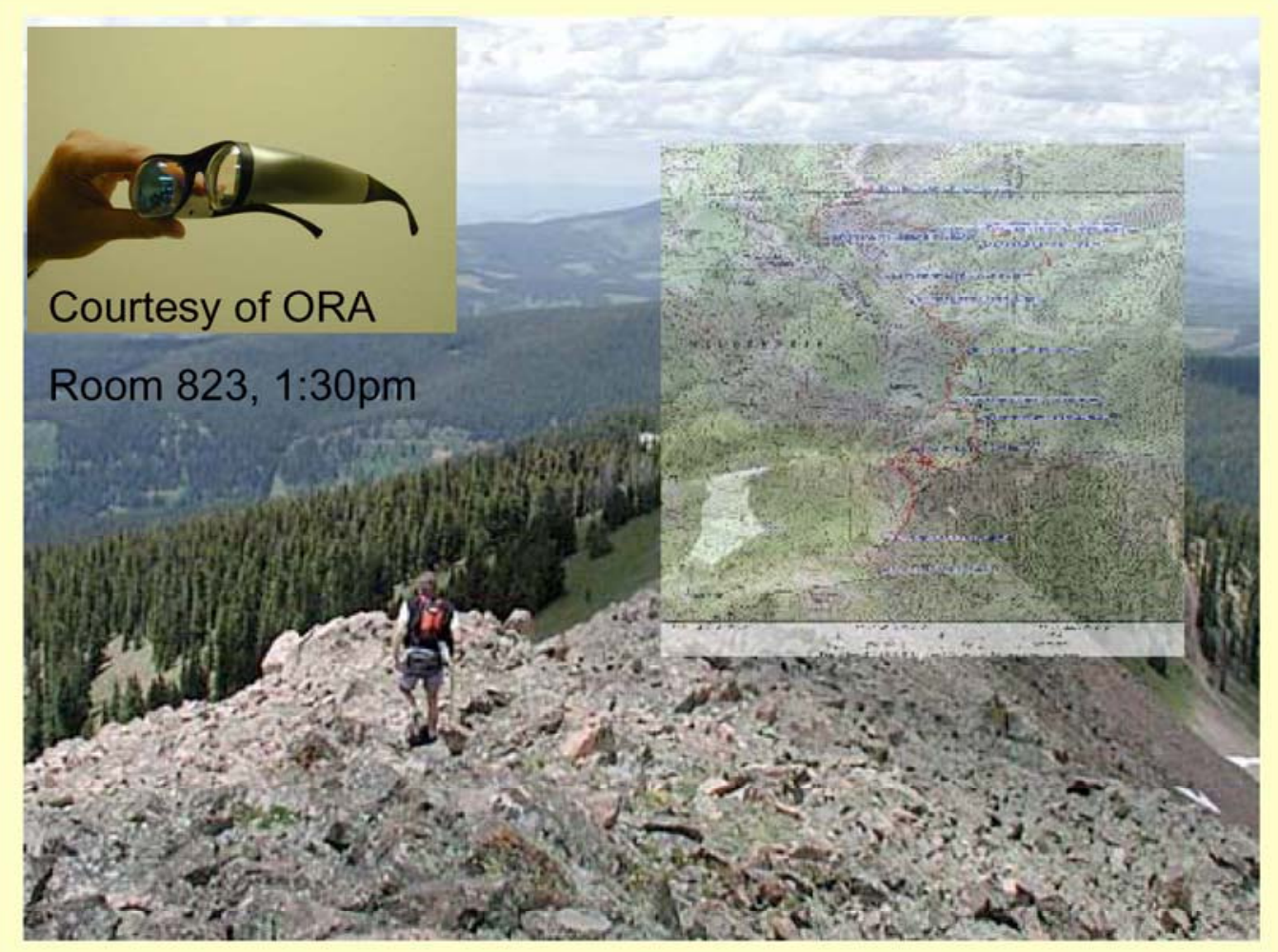

\section{Wearable Displays: A Range of Possibilities}

Their future lies in large part in their "seamless" integration with tangible interfaces around us

\section{Augmented Reality I Mixed Reality}

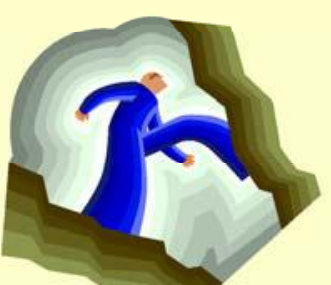

\section{Vs. Virtual Reality (full immersion)}




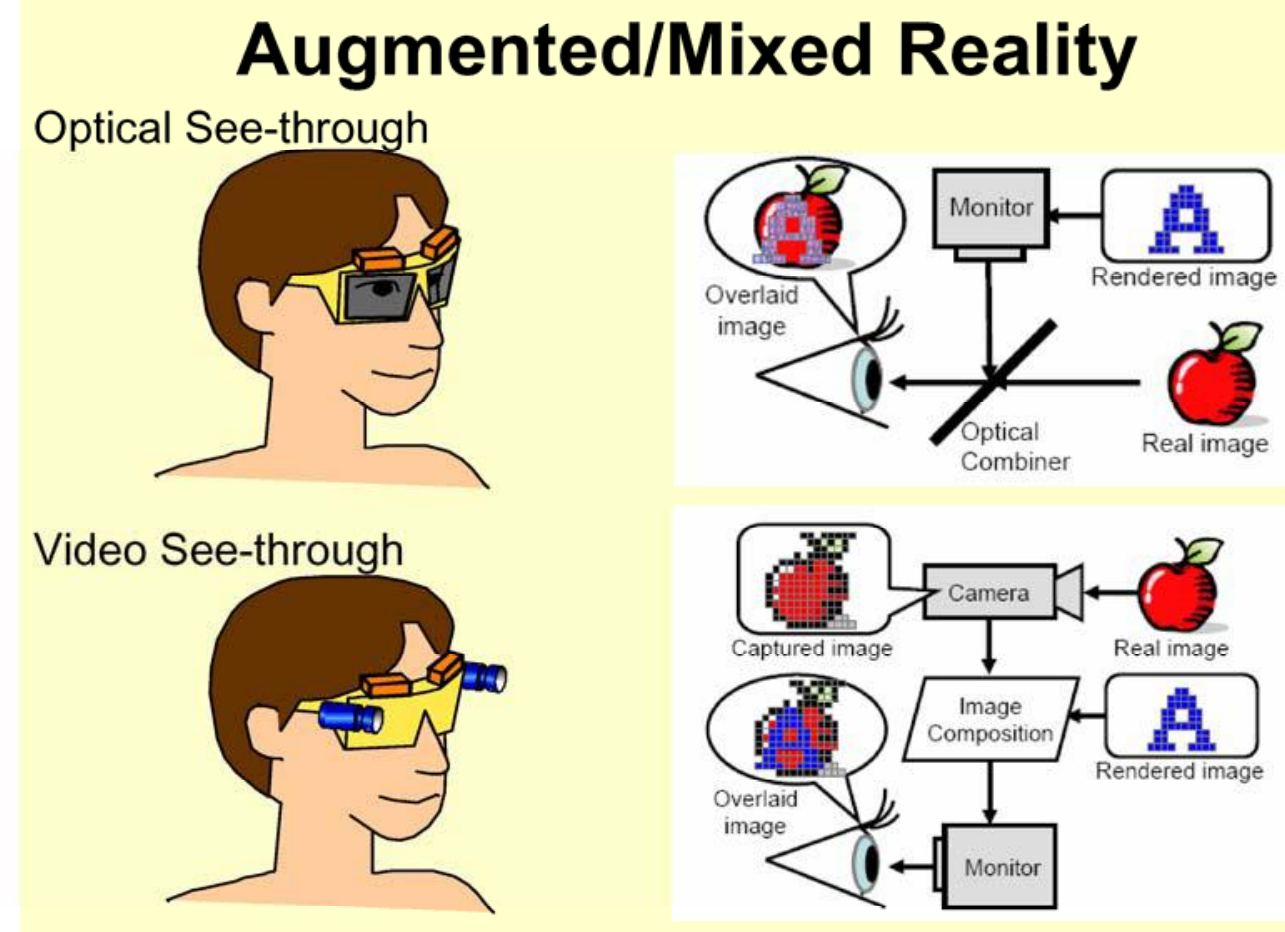

\section{Historical Notes}

First graphics-driven HWD was developed by Ivan Sutherland in the 1960s.
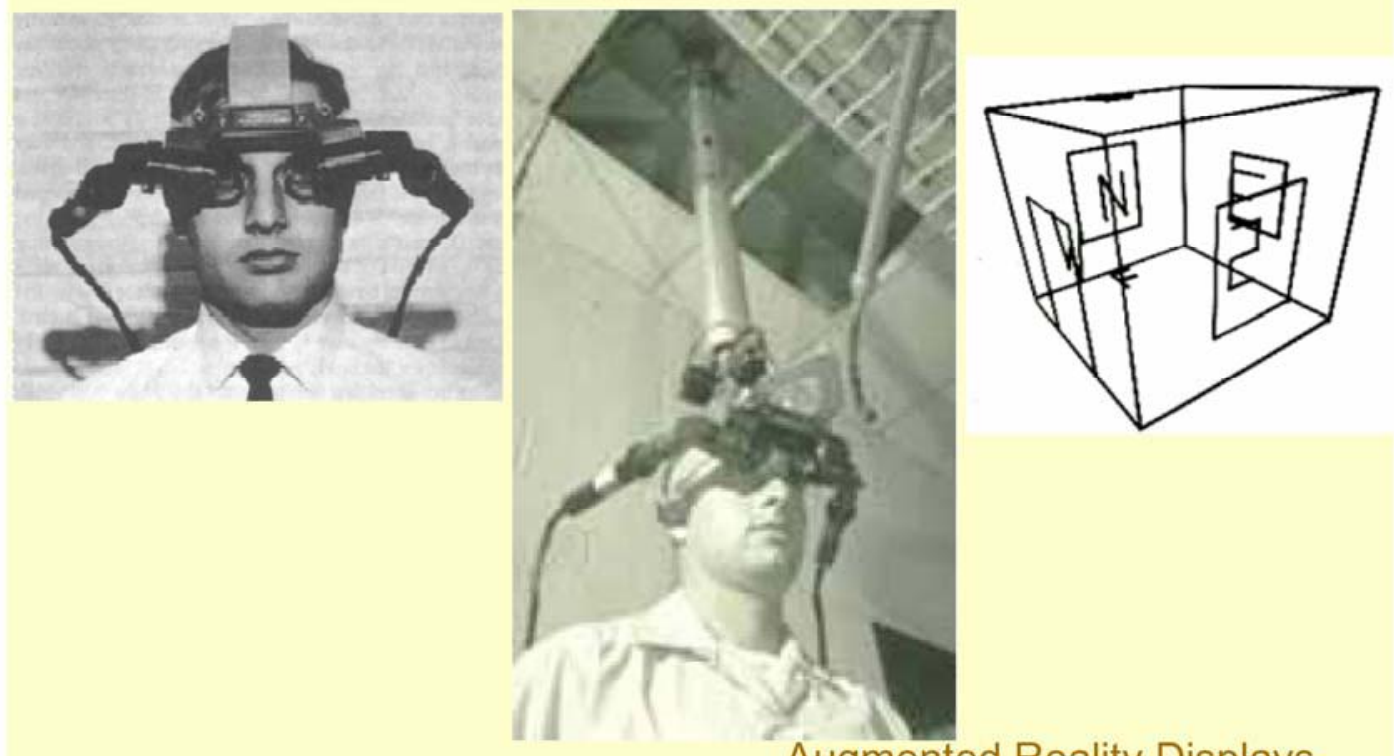

Augmented Reality Displays 


\section{Eardy (ijst?) stereoscopic V/ST-HWD}

- HMD-mounted stereo cameras with custom-designed lenses compensate for display distortion (Biocca \& Rolland, Presence 1998)
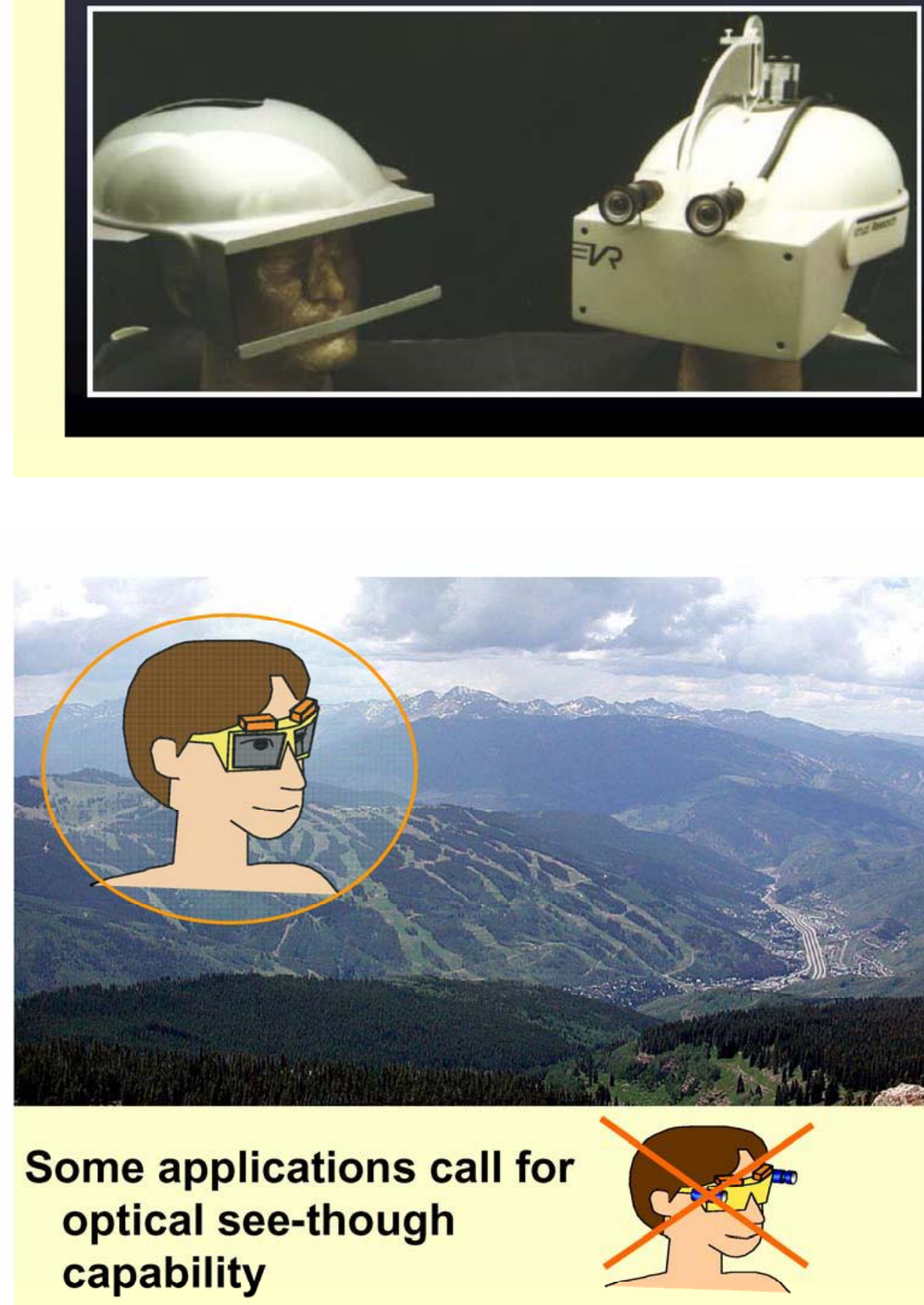


\section{Highlights from Past Development}

- U.S. Army first to fly a helmet-mounted sighting system on the Cobra helicopter.

- IHADSS (Integrated Helmet and Display Sighting System) was then deployed by the U.S. Army for the AH-64 Apache Helicopter.

IHADSS, while monocular, greatly contributed to the proliferation of all types of HMDs.

\section{The success of HWD design is most likely to occur when developed \\ - In the context of the users and \\ - Targeted at specific applications}

\section{A Main Design Trade-off}

FOV vs. Resolution - Currently limited by microdisplays Angle subtended by a pixel $=\frac{F O V}{\# \text { of pixels }} \quad$ Human eye 1 arcmin

\section{Approaches:}

1) High-resolution area of interest or inset

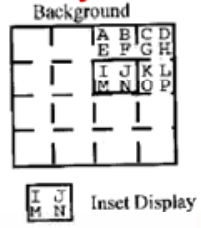

2) Partial binocular overlap ("Luning")

3) Optical tiling (Kaiser, Sensics)
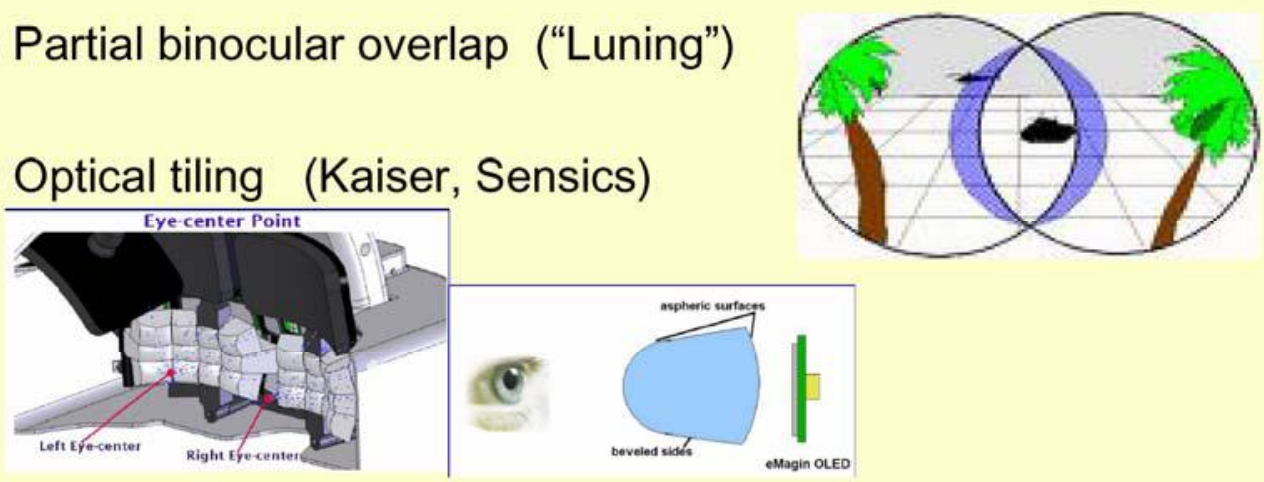

Recent developments by Sensics. 


\section{Driven by Medical Visualization: VRDA Tool "Virtual Reality Dynamic Anatomy"}

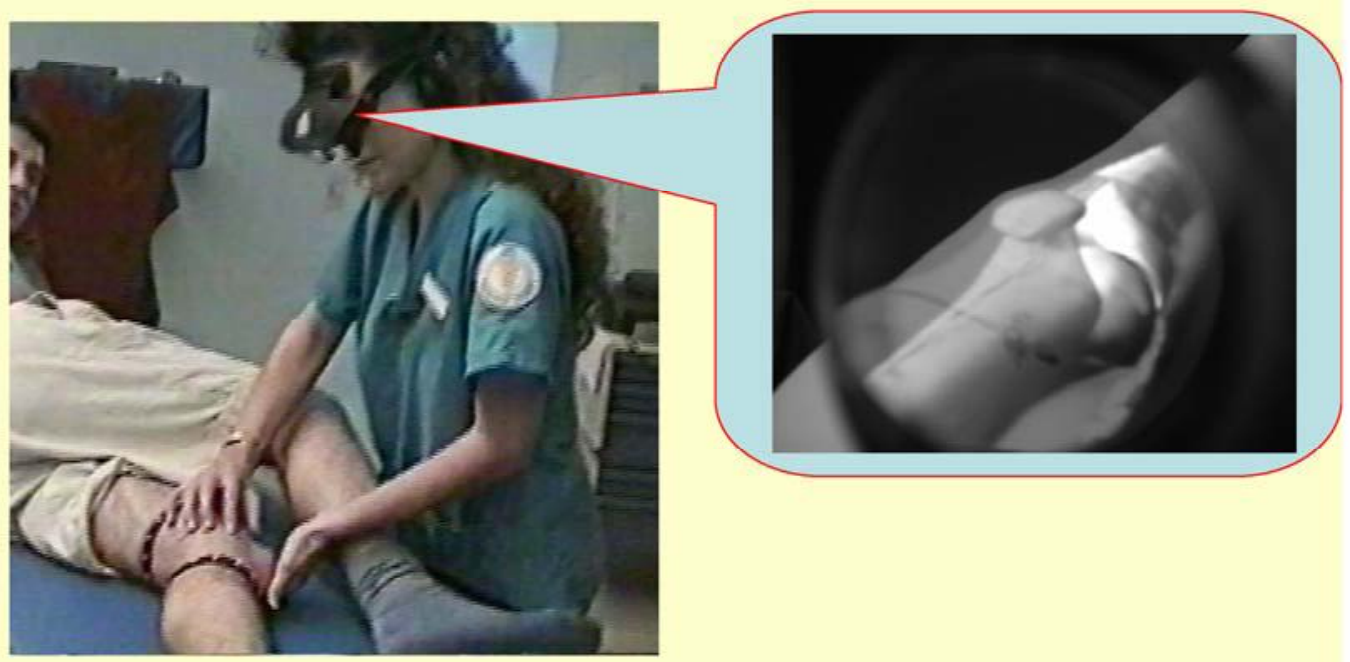

NIH - First Award 1997-2002

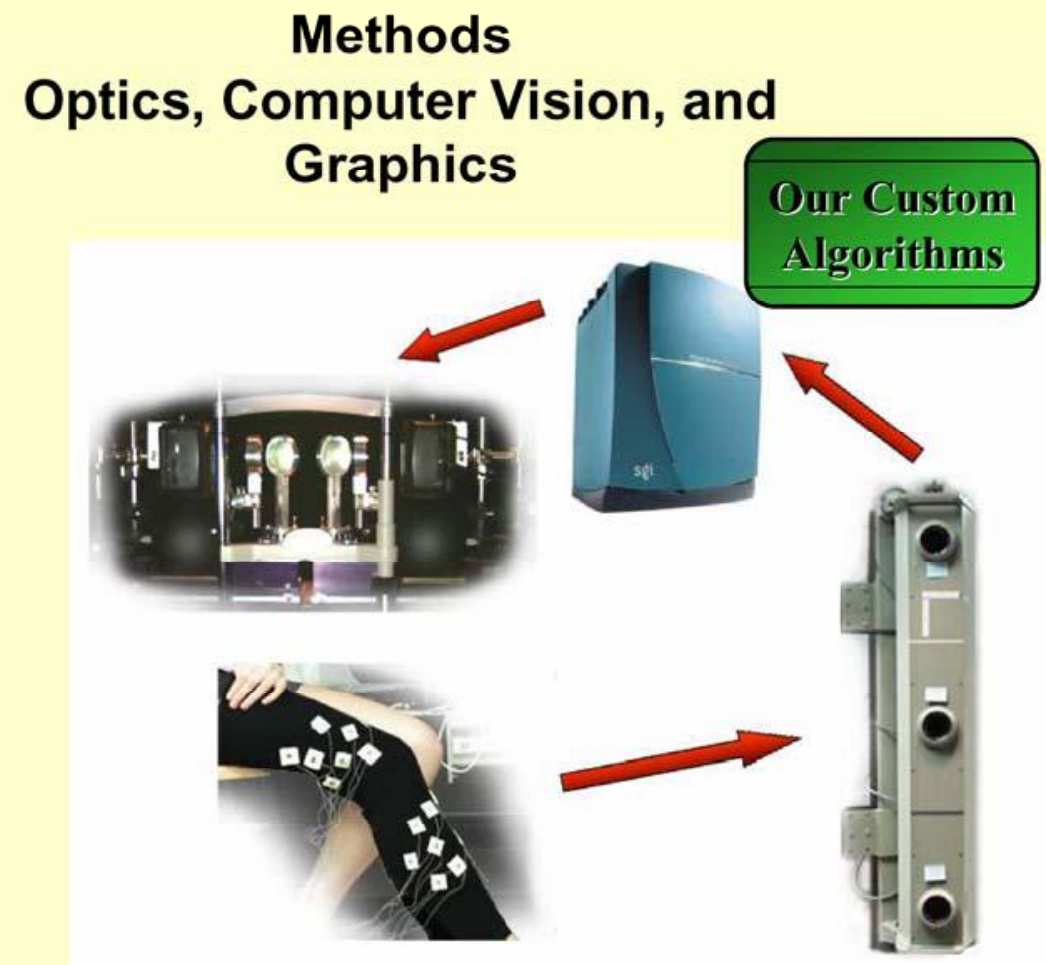


Development of a Kinematic Model of Joint Motion (Baillot, Rolland et al., 2000)

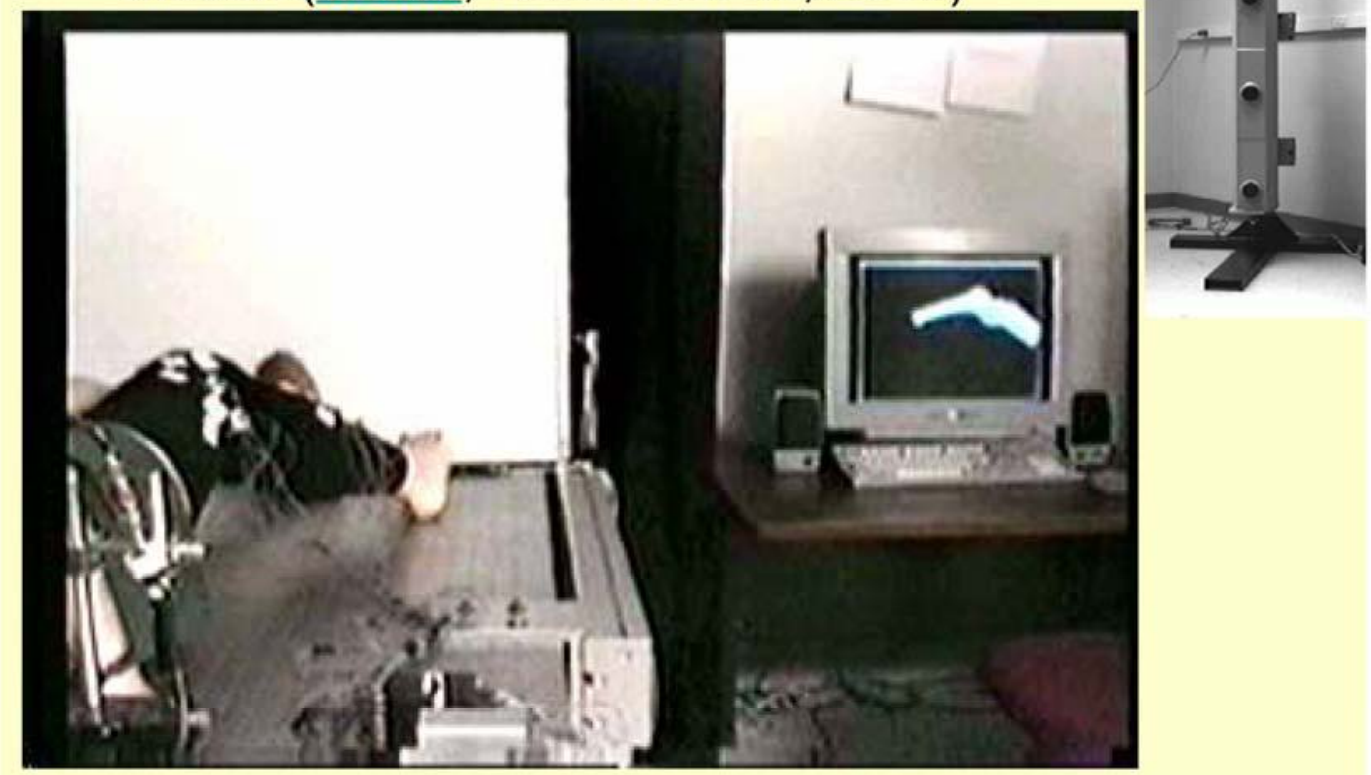

Early Feasibility Experiments

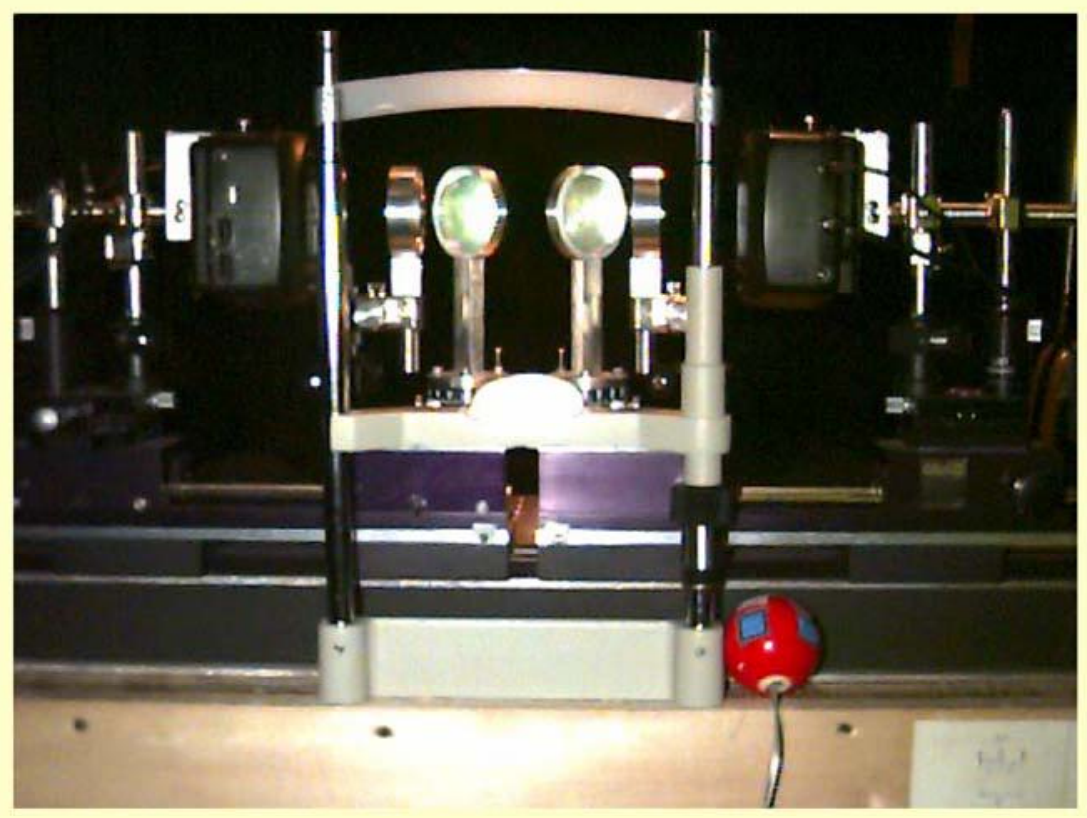




\section{First results in dynamic optical superimposition on}

an optical bench system

Featured in Scientific American, April 2002

Baillot et al., Presence 2000; Argotti et al., Computers \& Graphics 2002
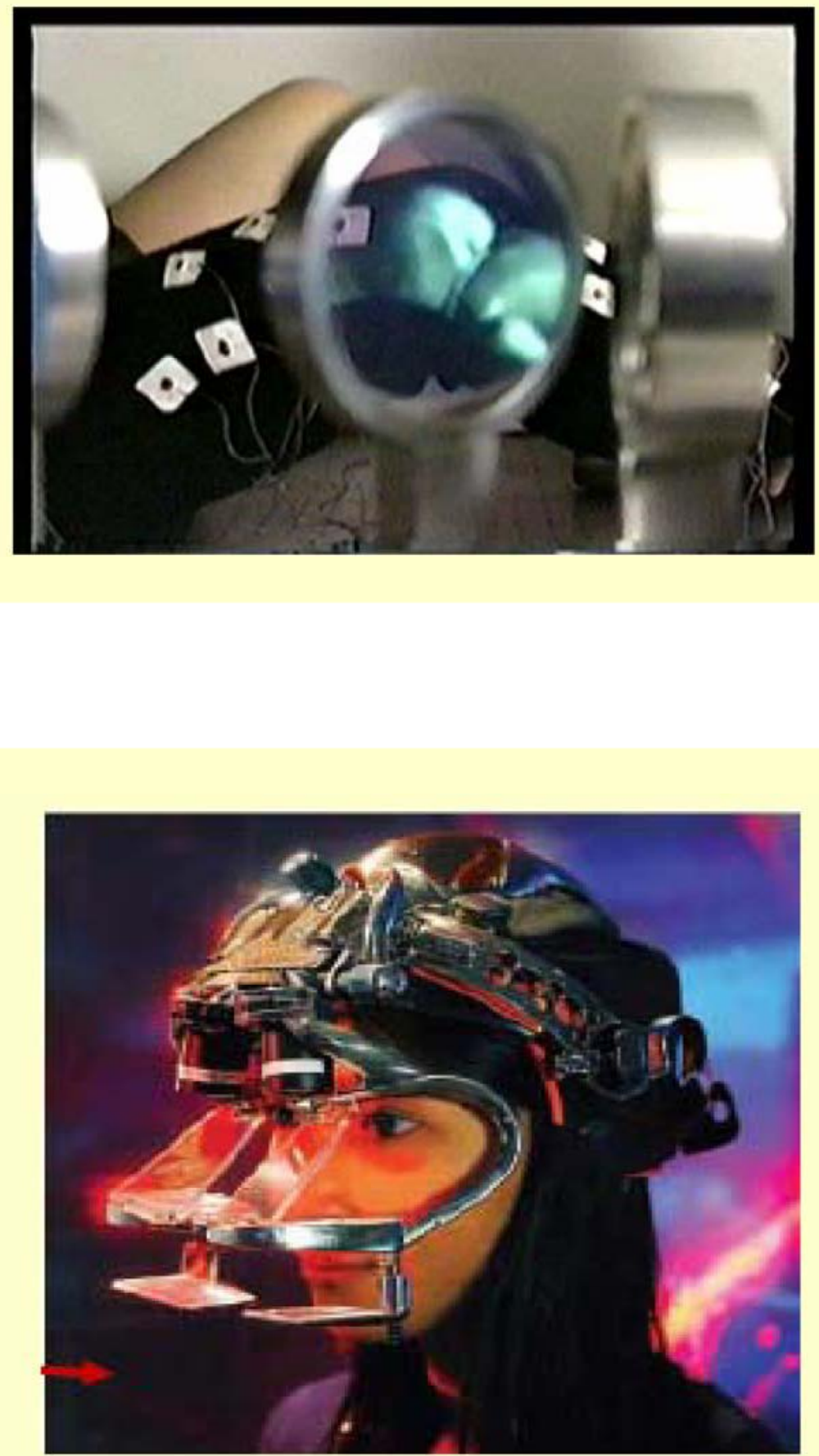


\section{Visualization (Head-Worn Displays)}

Cakmakci Ozan, and Jannick Rolland, Head-worn displays, IEEE/OSA Journal of Display Technology, 2(3) (September 2006).

C. Fidopiastis, L. Davis, J.Covelli, L. Nguyen, R. Martins, O. Cakmakci

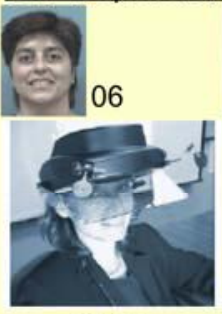

98-00
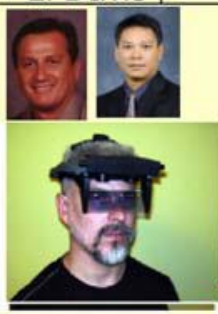

04

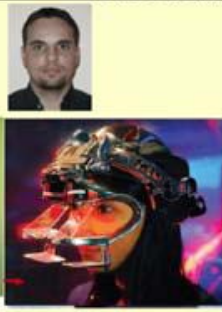

05-06

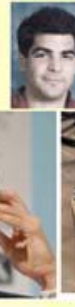

07

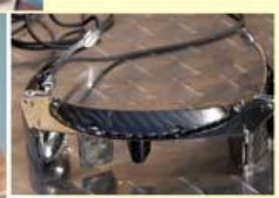

08

Students: F. Hamza-Lup, A. Santhanam
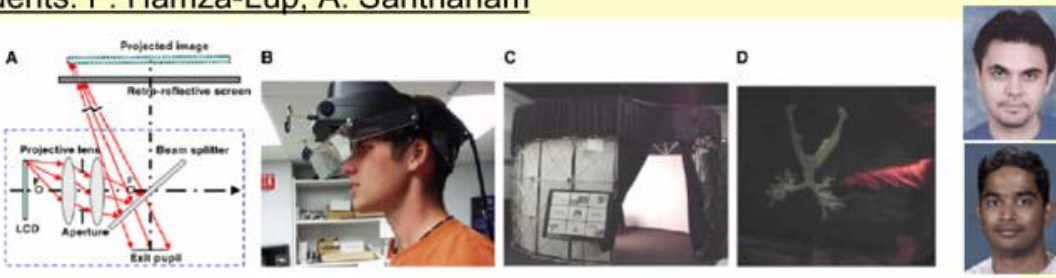

Fig. 5 HMPD in use in a deployable Augmented Reality Center (ARC): (A) Schematic of the HMPD optics; (B) user wearing a HMPD; (C) the ARC; and (D) user interacting with 3D models in the ARC. (View this an in color at www, dekker.com.)

\section{Eyepiece versus Projection HMDs}

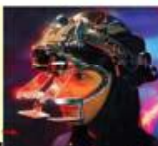

Eyepiece Optics (HWD)

Head Mounted Projection Display
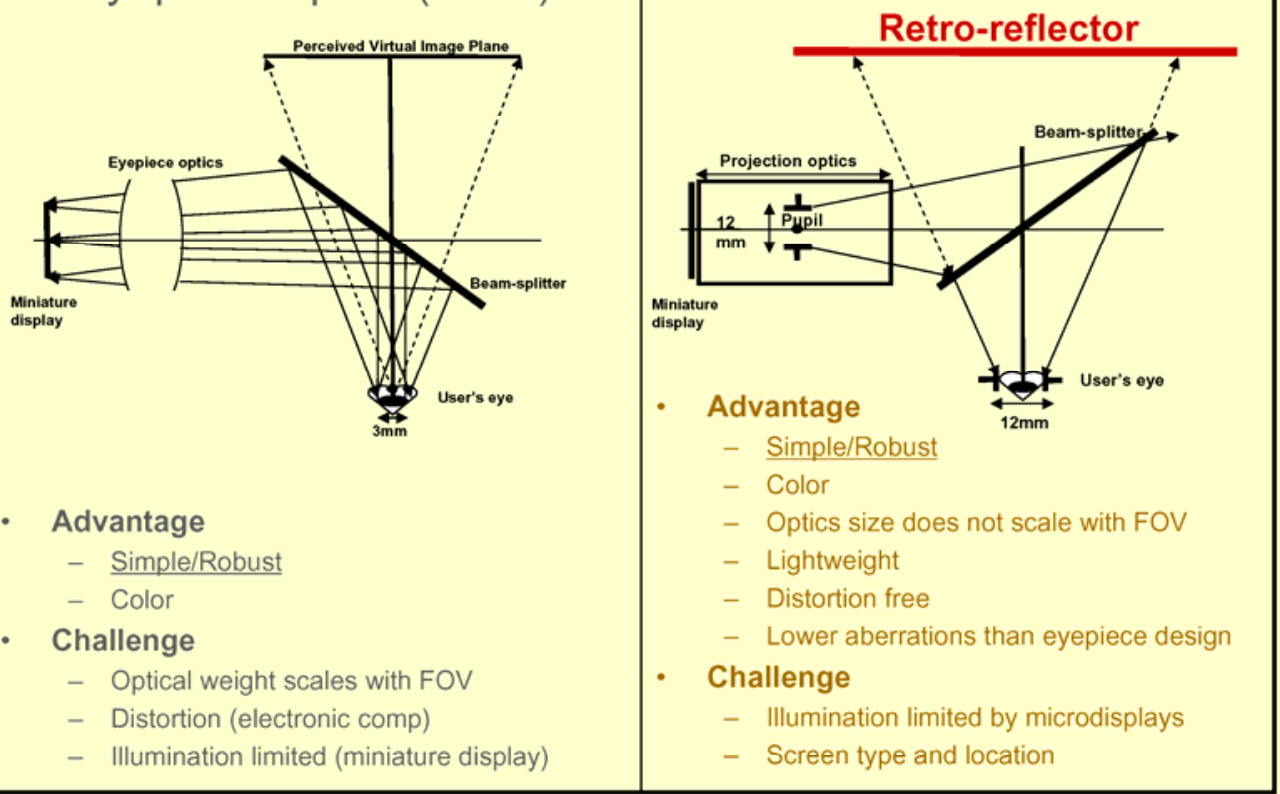

Advantage

- Simple/Robust

- Color

Challenge

- Optical weight scales with FOV

- Distortion (electronic comp)

- Illumination limited (miniature display) 


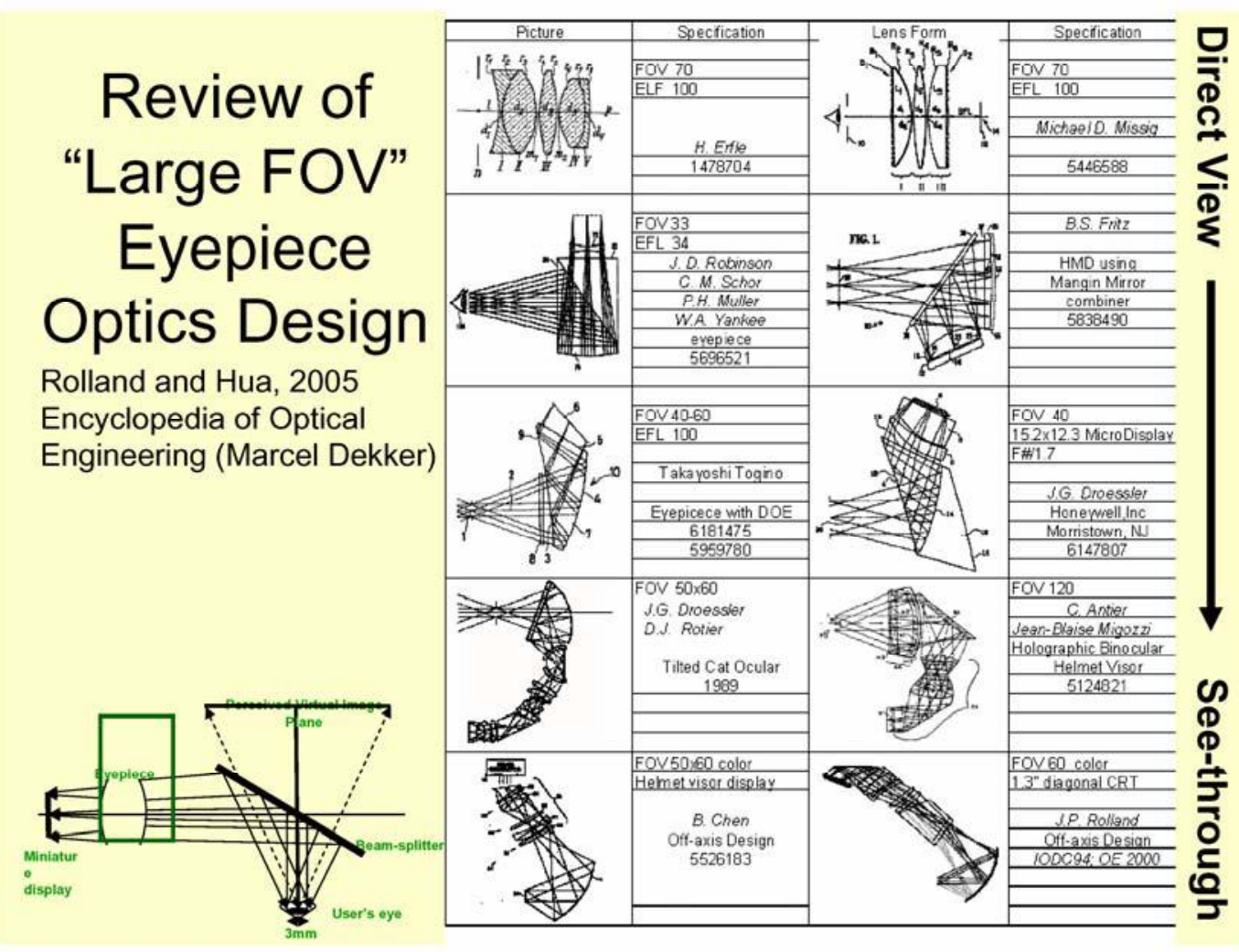

\section{Related Work}

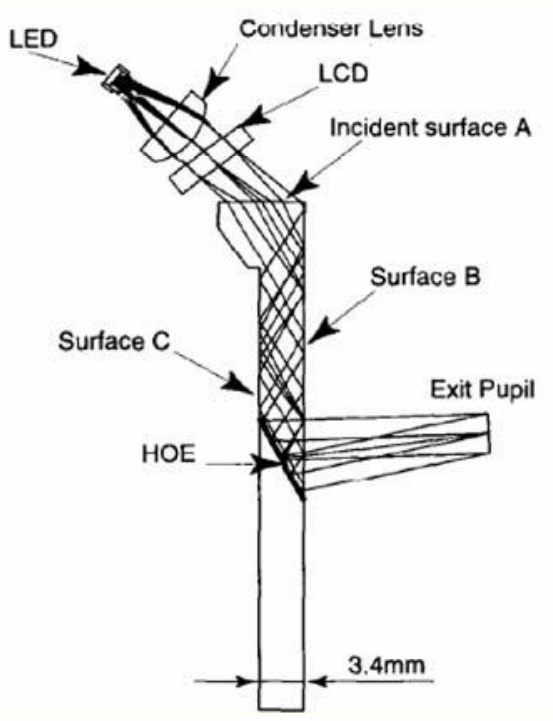

Kasai. Int. Symp. Wearable Computers '00.
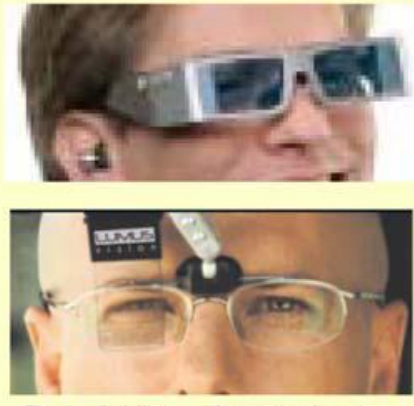

Resolution $\sim 2$ arcmins FOV $\sim 30$ degrees $10 \mathrm{~mm}$ pupil [Lumus]

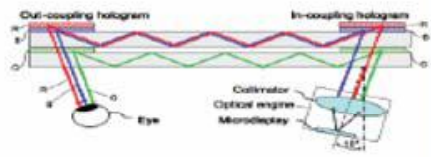

H. Mukawa et al. In Proc.

Society of Information Display, 2008. 


\section{AHMD (Advanced HMD) Ultrawide FOV, off-axis design}

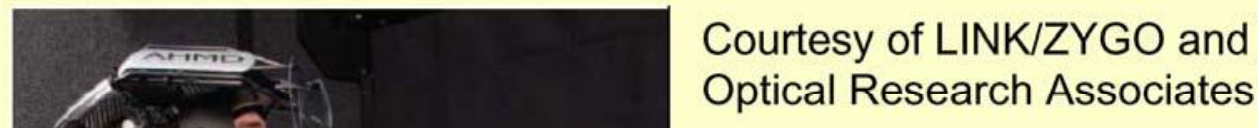

Early 2000

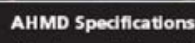

Helmet compatality HGU-56P, HGU-55:P

all sies

Center of gravity

Eye refet

Exit pupl

Batanced

$>50 \mathrm{~mm}$

$15 \mathrm{~mm}$

Transmisstivity

Field-of-view

$>60 \%$

Binocular overlap

$100^{\circ} \mathrm{H} \times 50^{\circ} \mathrm{V}$

Resolution

$30^{\circ}$

$1280 \times 1024$ per eye

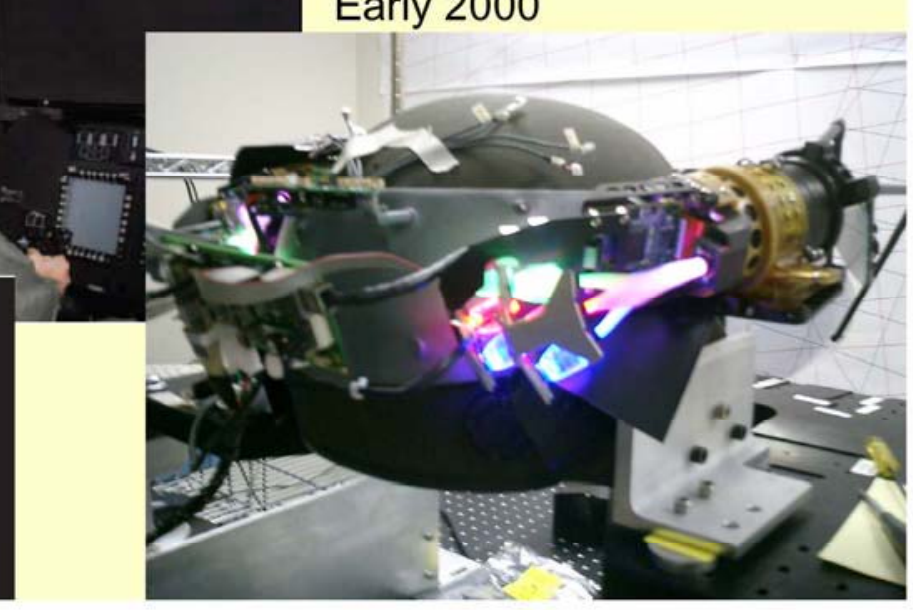

\section{Spatial Uniformity Behavior} with Freeform Bezier Shapes

Fournier et al., Appl. Opt. 47 (2008) \& OL 33(11) (2008)

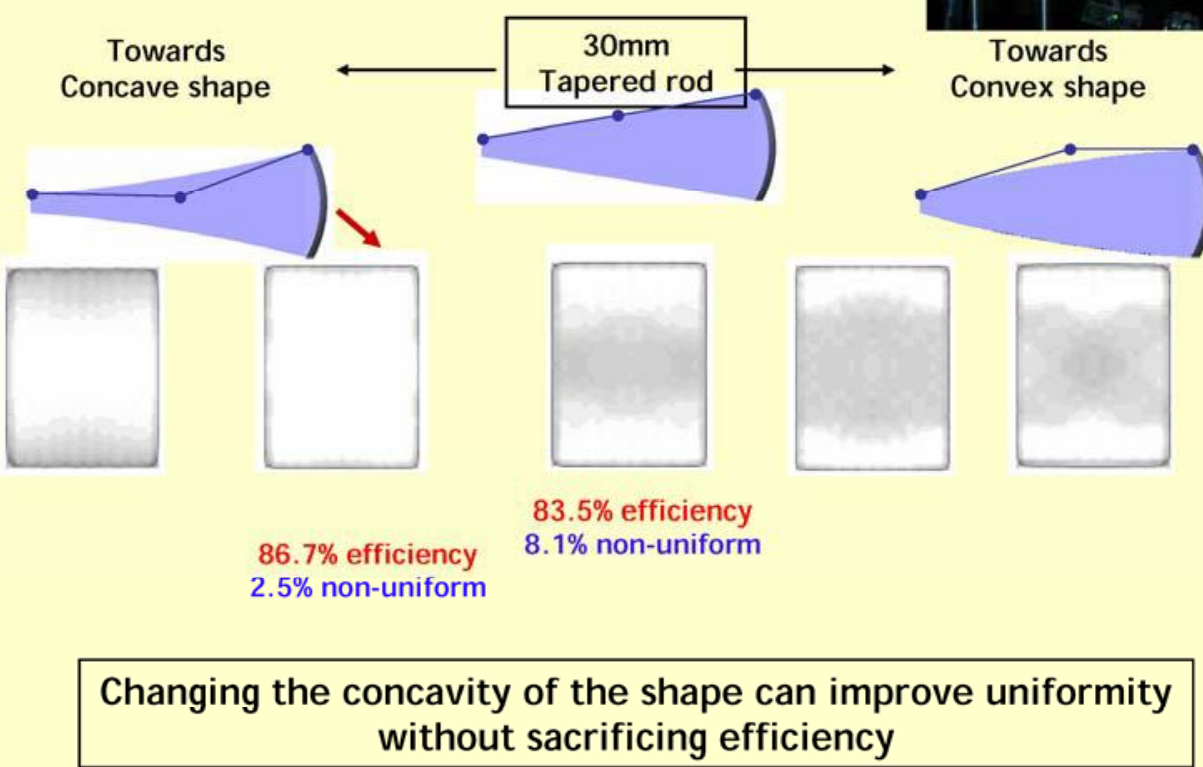

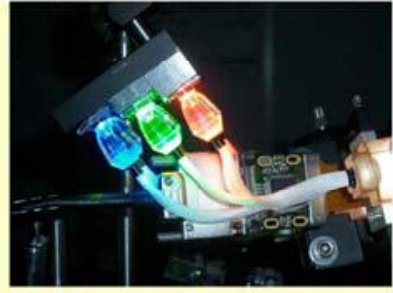

Towards 


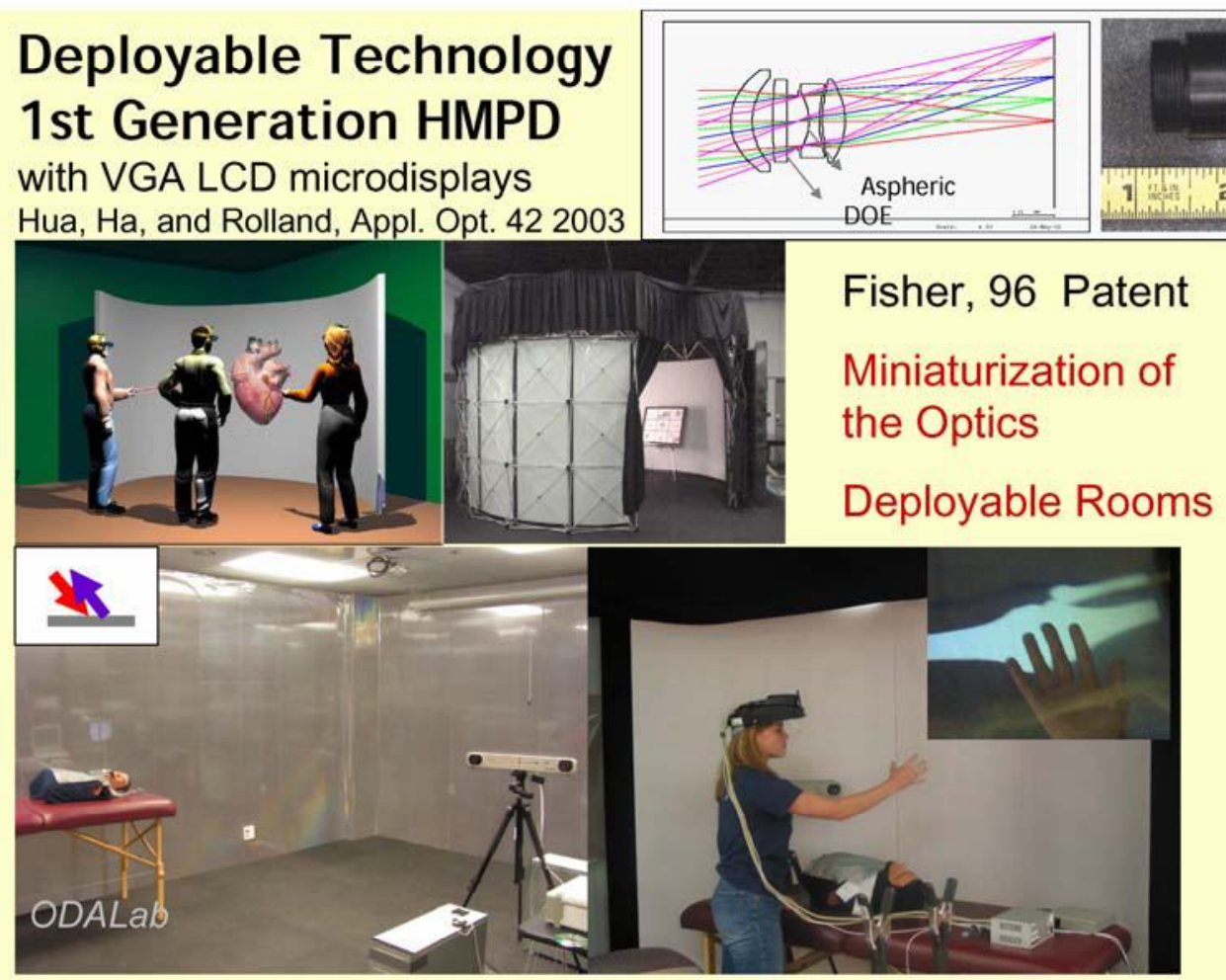

\section{D Visualization of the Upper Airway for Training Medics in Emergency Intubation Procedures}

Augmented Reality Visualization

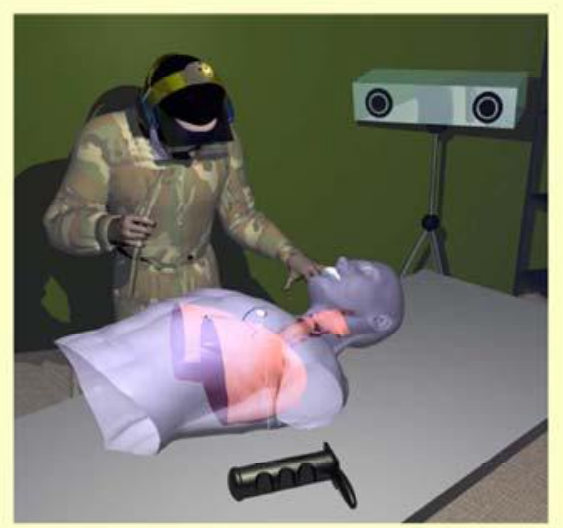

Lung Dynamics

Anand Santhanam, PhD 06
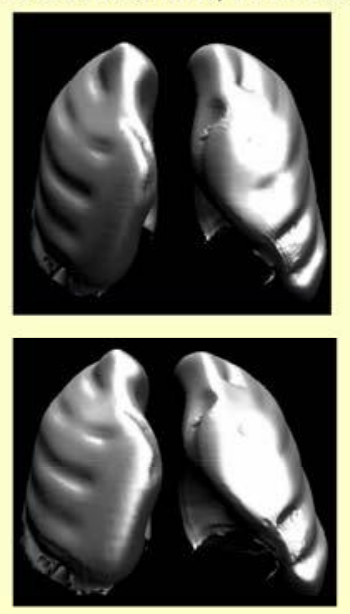


\section{Imaging: Extended Depth of Focus Needed in Catheters}

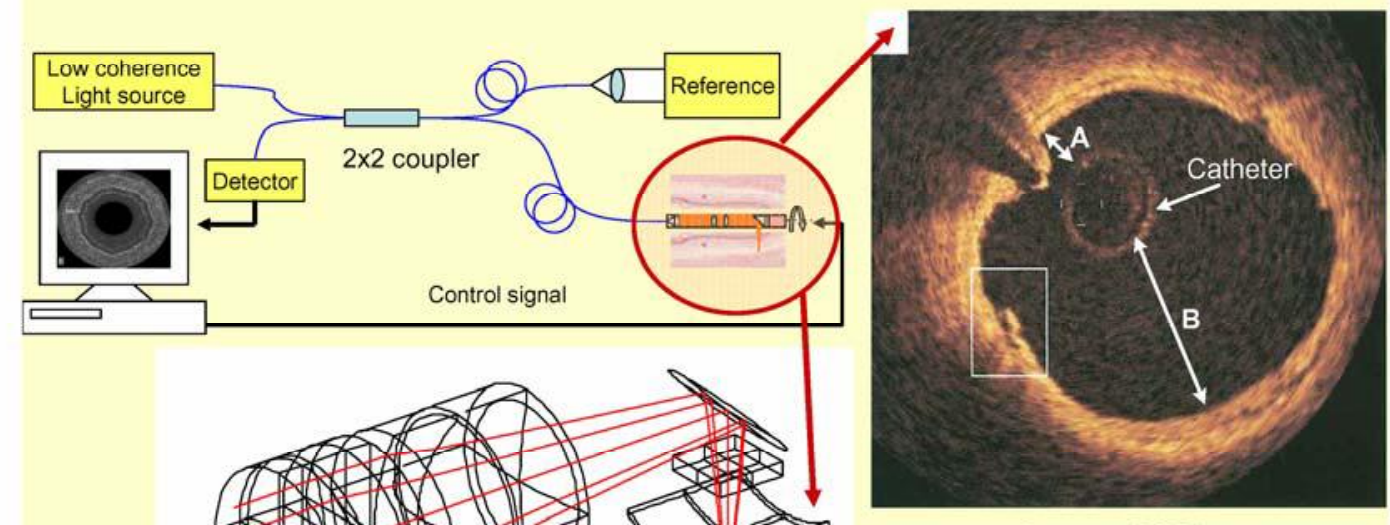

Coronary OCT image

J. Am. Coll. Cardiol. 2006;47:C69

Sheath

Required

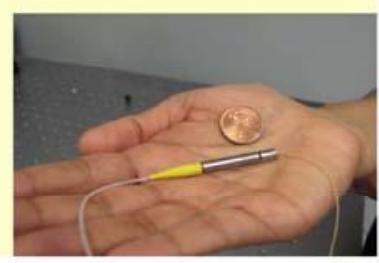

\section{PSF through Working Range}

Meemon et al., AO 2008
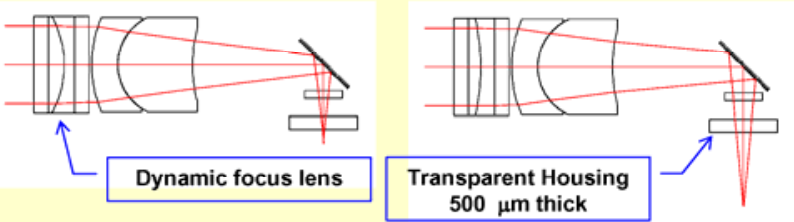

Target $\rightarrow$ Strehl ratio $>0.8$

\section{Strehl Ratio}

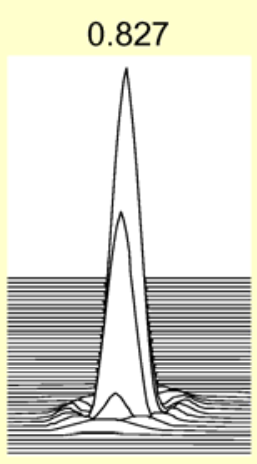

$\sim 0.5 \mathrm{~mm}$

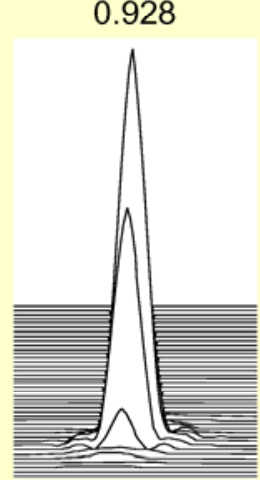

$\sim 1.5 \mathrm{~mm}$

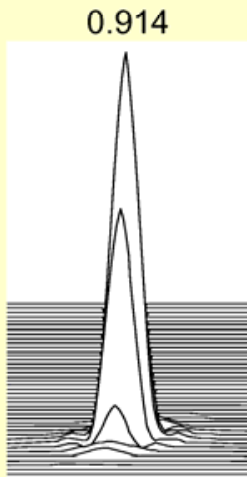

$\sim 3 \mathrm{~mm}$

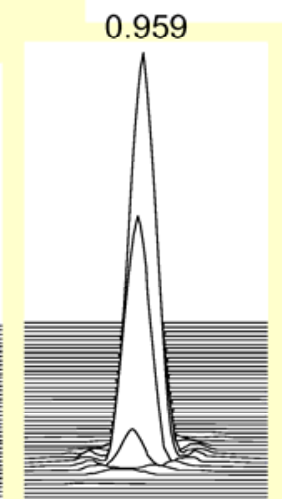

$\sim 4.5 \mathrm{~mm}$

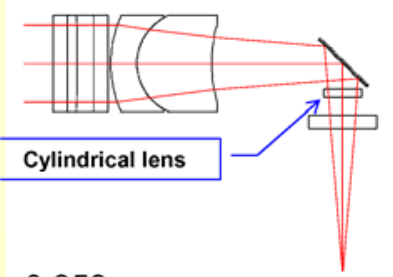

0.889

Distance from focal point to the outer surface of the exit window

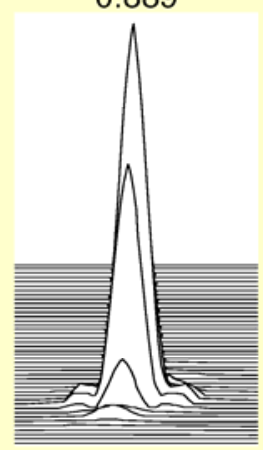

$\sim 5.5 \mathrm{~mm}$ 


\section{Bessel Beam vs. Conventional}
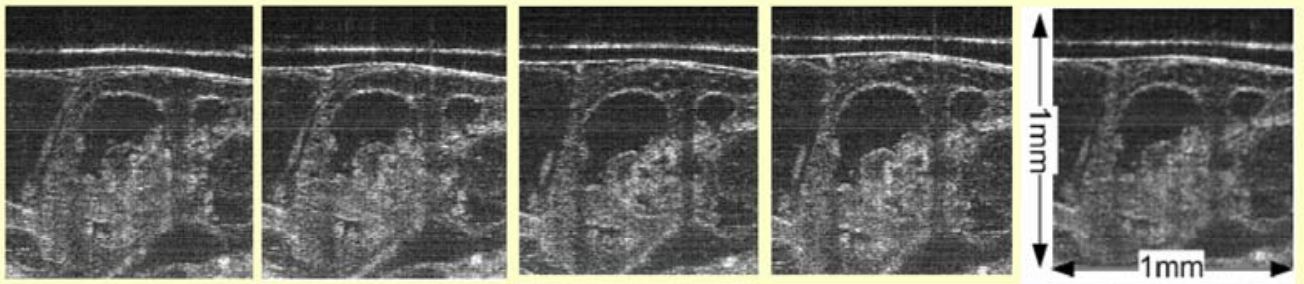

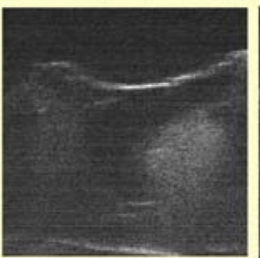

$d=-2 \mathrm{~mm}$

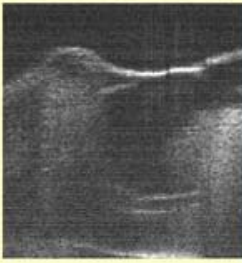

$d=-1 \mathrm{~mm}$

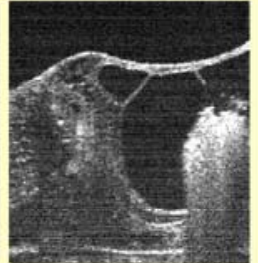

$d=0 \mathrm{~mm}$

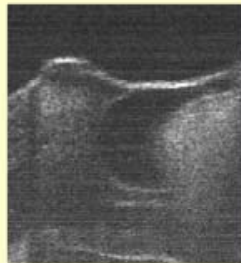

$d=1 \mathrm{~mm}$

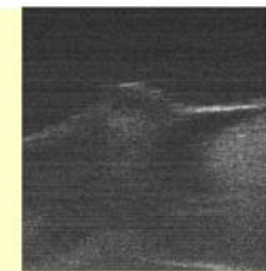

$d=\mathbf{2} \mathbf{m m}$

First Images of biological tissue acquired with a microlens axicon in a double pass OCT : Images of African frog (Xenopus Laevis) tadpole located at relative axial distances $\boldsymbol{d}$ from each medial position of its depth of focus.

\section{2 deg. Lens / $8 g$ per eye}
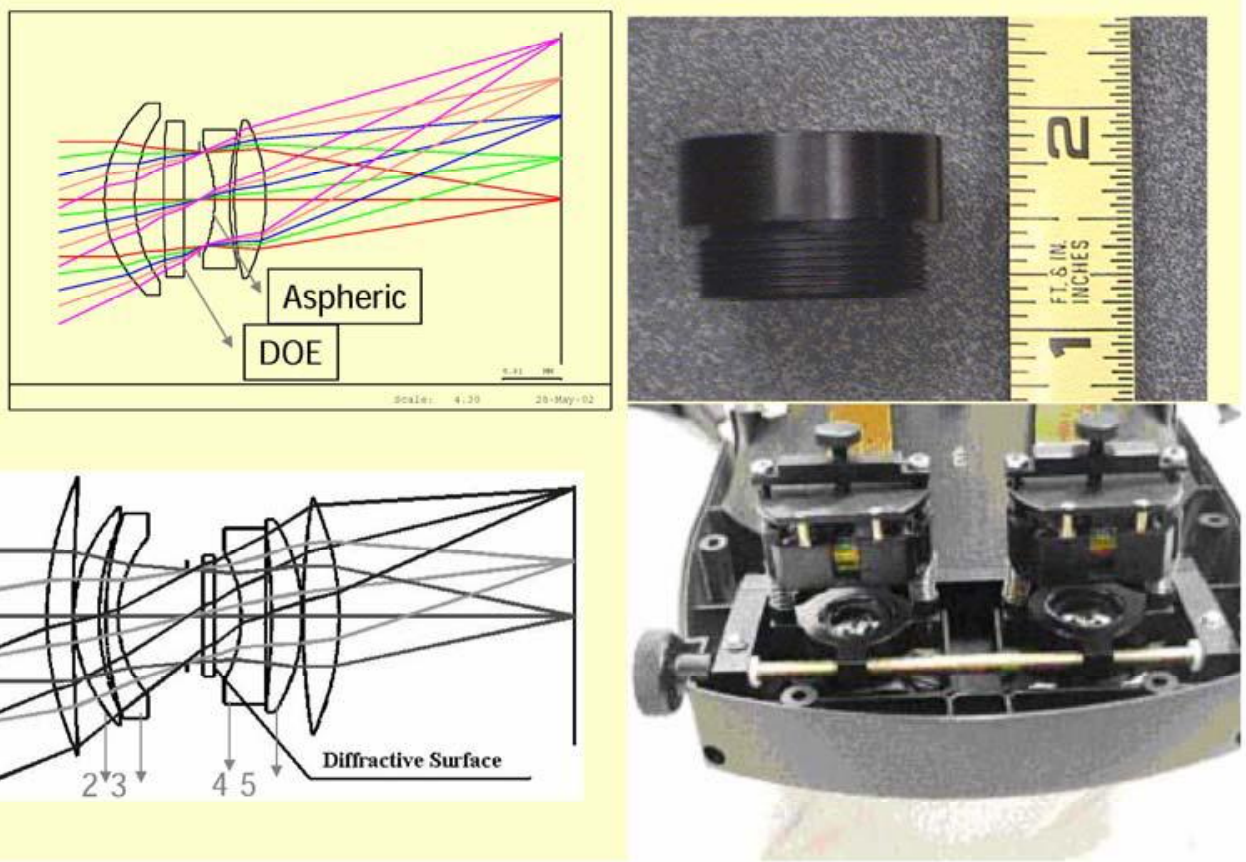

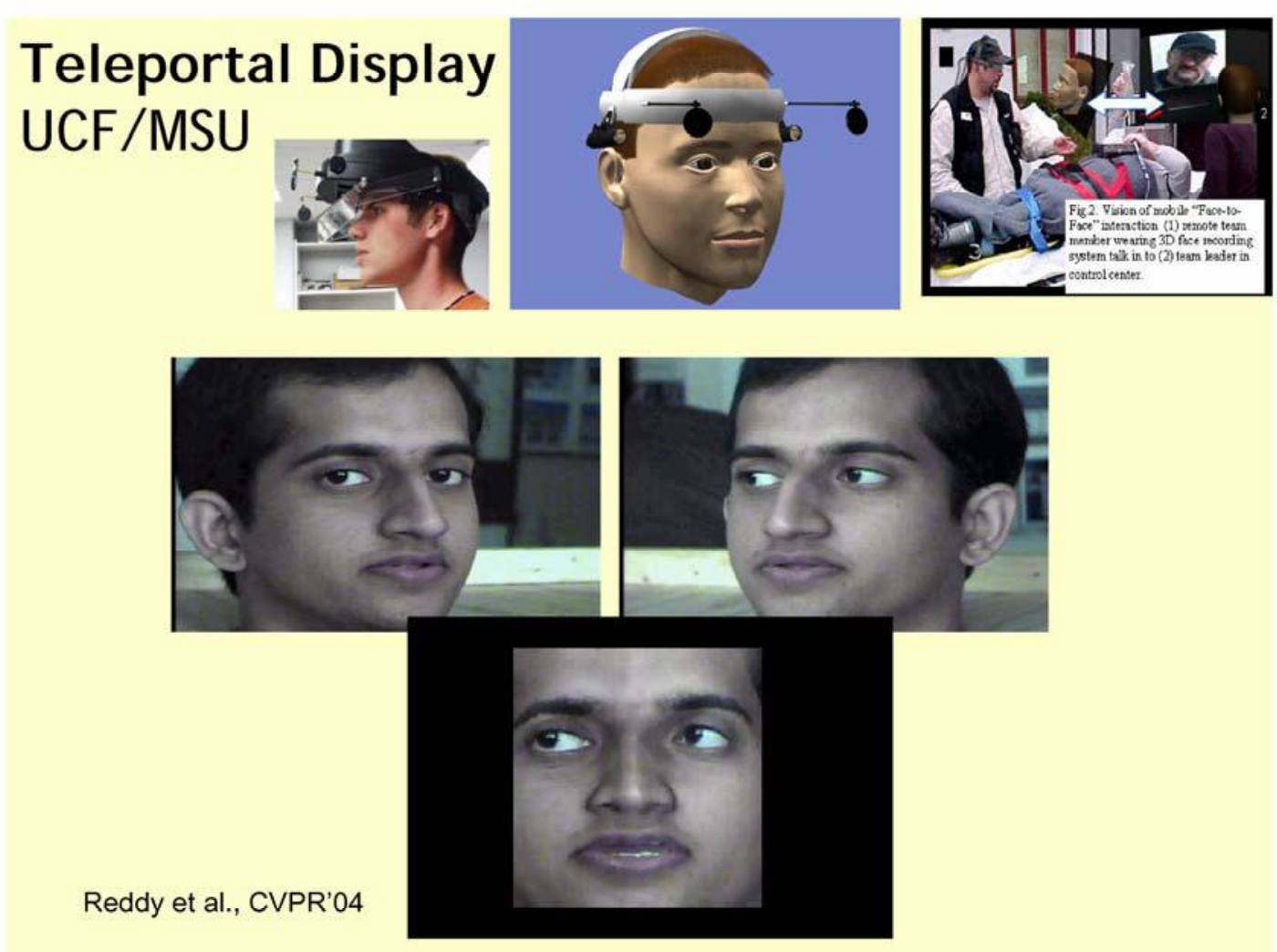

\section{$42^{\circ}$ FOV HMPD}

Lightweight 595 grams - $2^{\text {nd }}$ Generation HMPD using 800x600 OLED

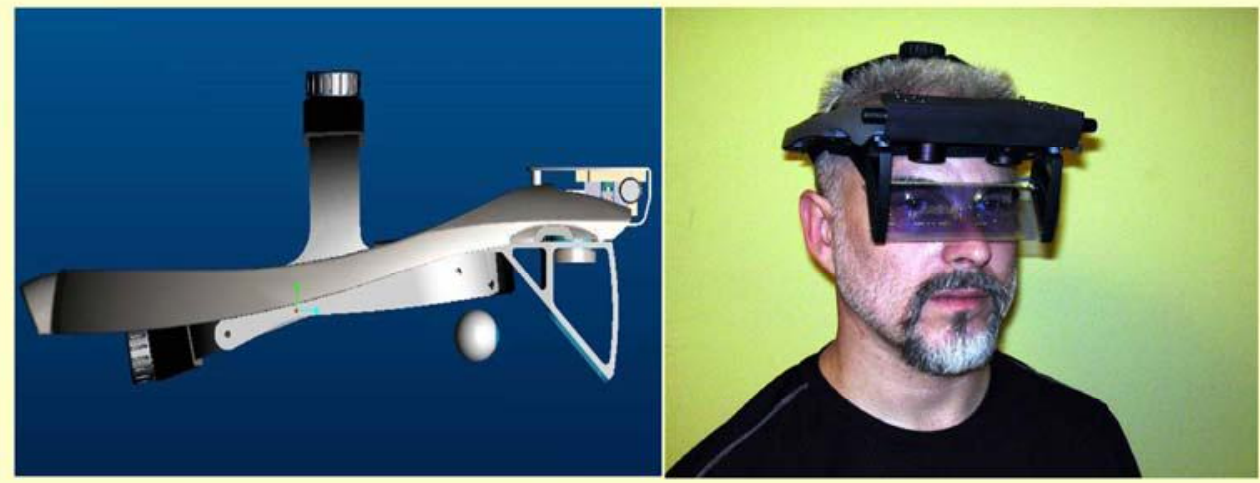

Optical Design done in the ODALab and

HMPD Optomechanical design done by Nvis Corporation under SBIR program 2004-2005 with the US ARMY 


\section{M-HMPD - Fabric-free, Mobile}

Martins, Optics Express 15(22), 2007

See-through, Outdoor $42^{\circ} \mathrm{FOV}$
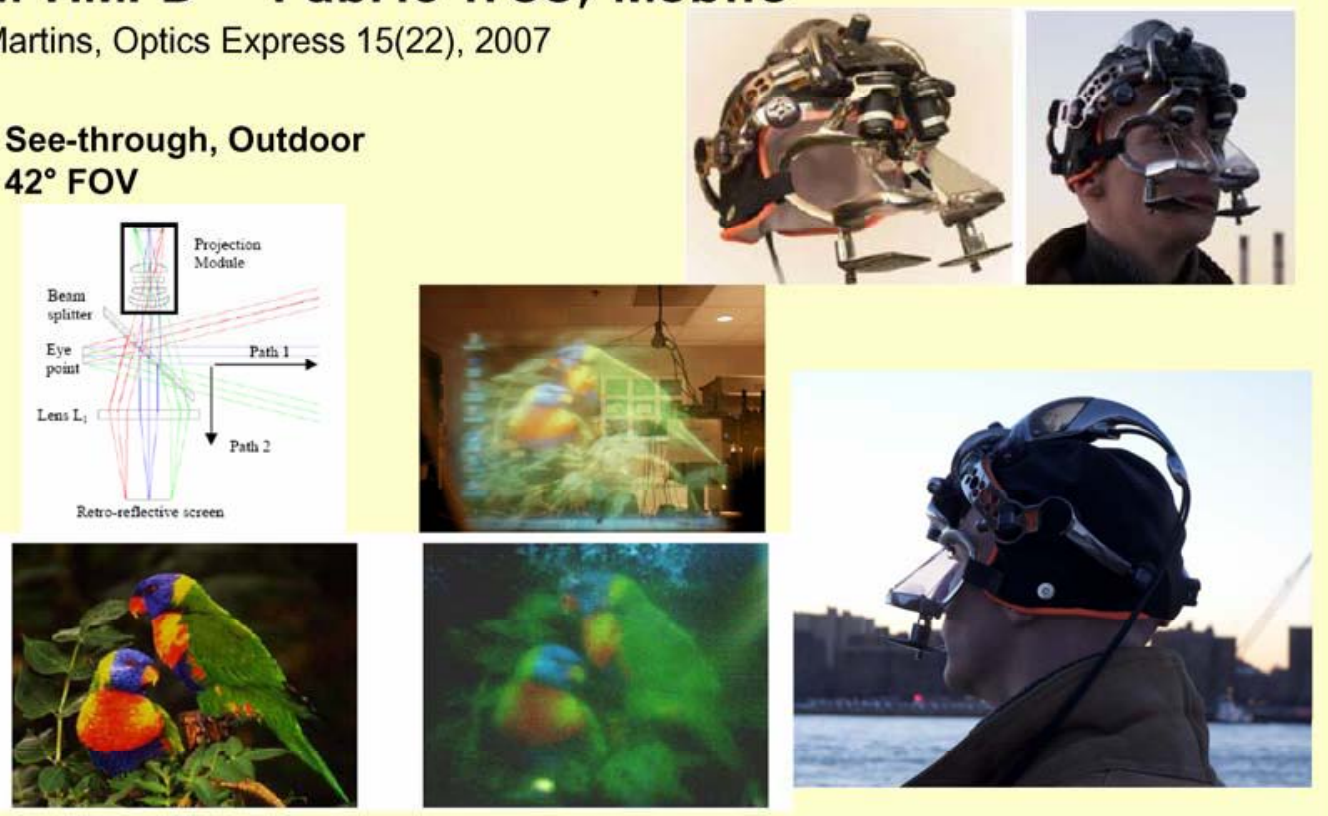

\section{A recent experiment with the MD Anderson Cancer Center Orlando to appear in JDT, Dec08}




\section{Comparison of the ARC system with the 2D display system}

\section{To appear in Special Issue of JDT, Dec 08}

\begin{tabular}{|l|c|c|c|c|c|c|}
\hline \multirow{2}{*}{ Subject } & \multicolumn{2}{|c|}{$\begin{array}{c}\text { Average time (sec) } \\
\text { Experiment 1 }\end{array}$} & \multicolumn{2}{c|}{$\begin{array}{c}\text { Average time (sec) } \\
\text { Experiment 2 }\end{array}$} & \multicolumn{2}{c|}{$\begin{array}{c}\text { Average time (sec) } \\
\text { Experiment 3 }\end{array}$} \\
\cline { 2 - 7 } & ARC & $\begin{array}{c}\text { 2D } \\
\text { monitor }\end{array}$ & ARC & $\begin{array}{c}\text { 2D } \\
\text { monitor }\end{array}$ & ARC & $\begin{array}{c}\text { 2D } \\
\text { monitor }\end{array}$ \\
\hline Expert 1 & 0 & 2.55 & 0.75 & 11.05 & 1.05 & 13.05 \\
\hline Expert 2 & 0 & 0.95 & 1.05 & 8.95 & 0.95 & 11.0 \\
\hline Expert 3 & 0.45 & 4.05 & 0.95 & 12.05 & 1.55 & 15.05 \\
\hline Expert 4 & 0 & 3.95 & 0.55 & 14.95 & 1.05 & 14.05 \\
\hline Expert 5 & 0.55 & 2.55 & 1.45 & 8.0 & 0.9 & 16.0 \\
\hline Expert 6 & 0 & 3.45 & 1.40 & 9.0 & 1.55 & 13.0 \\
\hline Average & $\mathbf{0 . 2}$ & $\mathbf{2 . 9}$ & $\mathbf{1 . 0}$ & $\mathbf{1 0 . 7}$ & 1.2 & 13.7 \\
\hline
\end{tabular}

The individual dose beams are delivered to a patient in 30-40 seconds, Thus, a 10 second delay in decision making is highly significant

\section{Visualization (Head-Worn Displays)}

Cakmakci Ozan, and Jannick Rolland, Head-worn displays, IEEE/OSA Journal of Display Technology, 2(3) (September 2006).

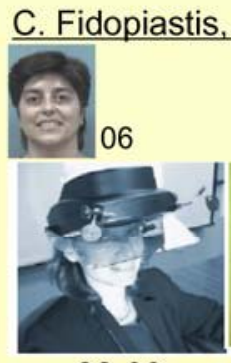

$98-00$

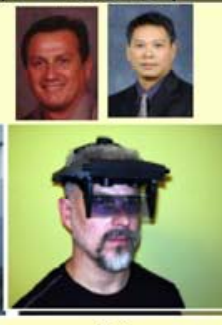

04 J.Covelli, L. Nguyen, R. Martins, O. Cakmakci

Students: F. Hamza-Lup, A. Santhanam
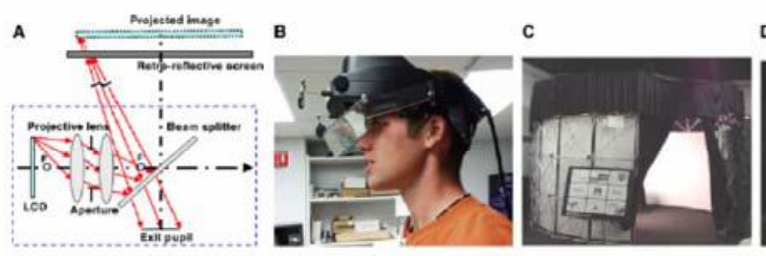

D

Fig. 5 HMPD in use in a deployable Augmented Reality Center (ARC): (A) Schematic of the HMPD opties; (B) user wearing a HMPD; (C) the ARC; and (D) user interacting with 3D models in the ARC. (View this an in color at www.dekker.com.) 


\section{FREE FORM OPTICS}

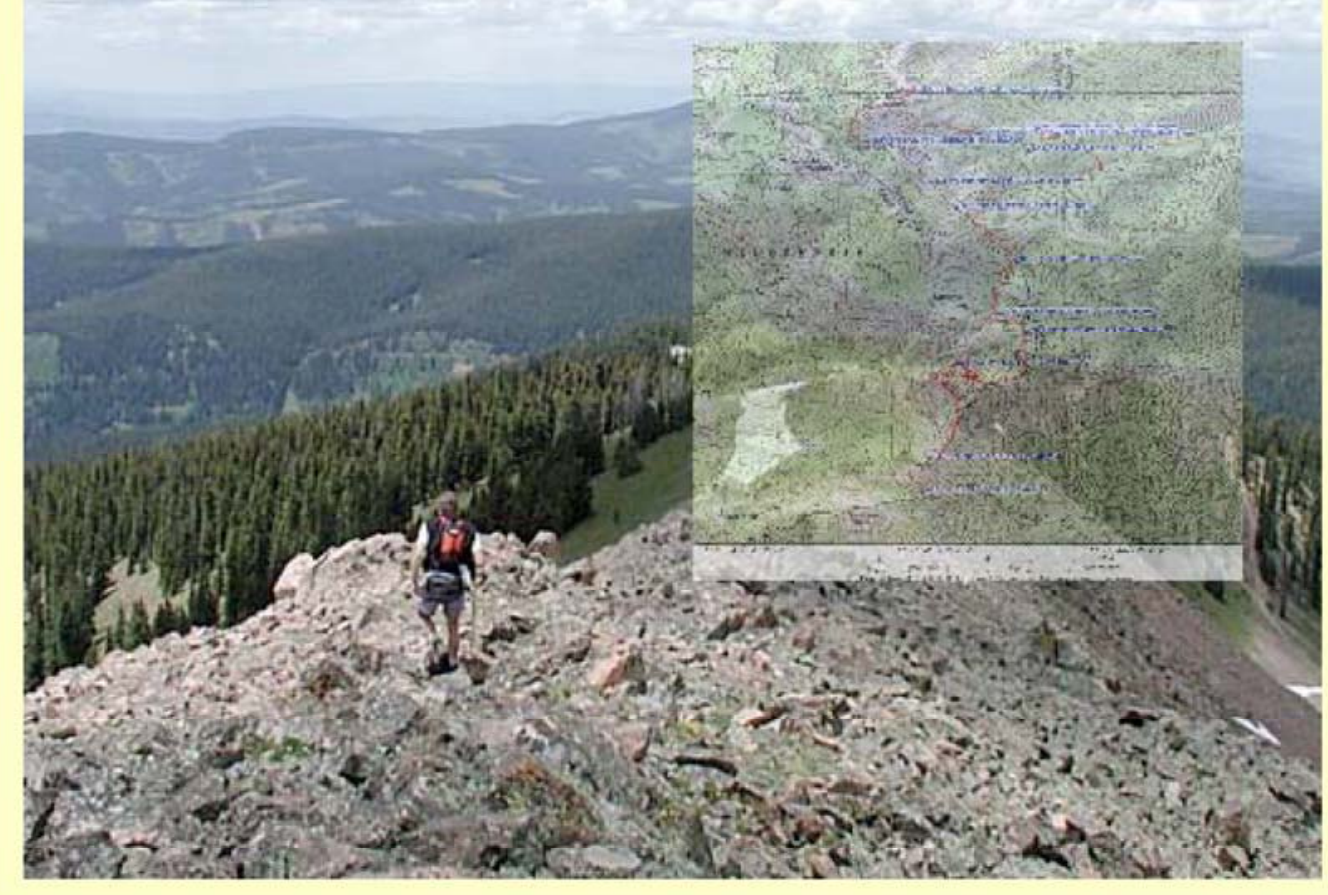

\section{Eyeglass Display Ozan Cakmakci, Kidger Scholarship 05}

Cakmakci \& Rolland, Journal of Display Technology, (2006).
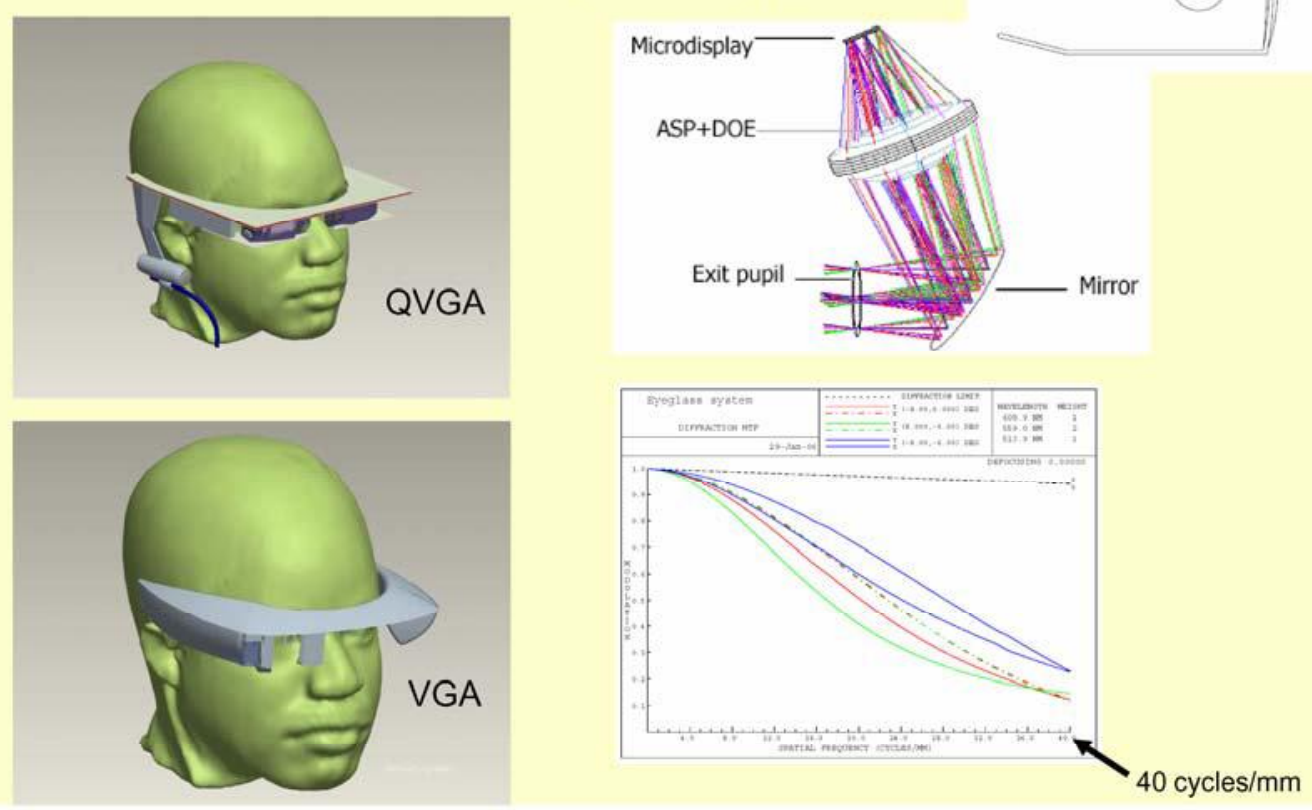


\section{Dual-element Solution}

Cakmakci \& Rolland, OL 32(11), 2007

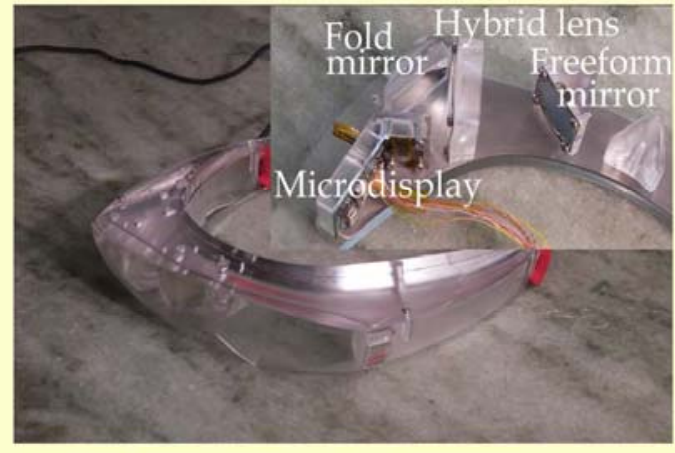

Field of view: up to 25 FOV diagonal Resolution: $~ 1.5$ arcminutes Exit pupil size: up to $12 \mathrm{~mm}$ Eye clearance: $>15 \mathrm{~mm}$ Distortion: $<4 \%$

Wavelengths: $450-650 \mathrm{~nm}$

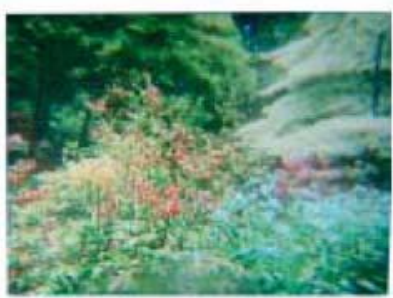

(a)

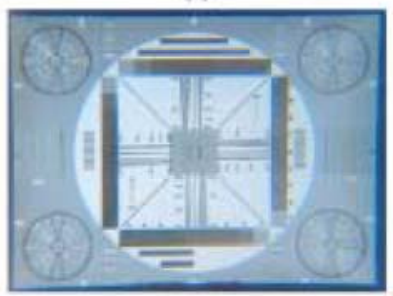

(b)

Fig. 2 (Color onlina) Photograph through the fabricated dual-element system of (a) a color target and (b) a black and white target.

\section{We Propose to Design Freeform Optical Surfaces whose Representations use Local Basis Functions (as Opposed to Global Polynomials)}

- An optical surface can be represented as a sum of basis functions

$$
z(x, y)=\sum \phi_{i}(x, y) w_{i}
$$

- In matrix form

$$
z=\Phi w
$$

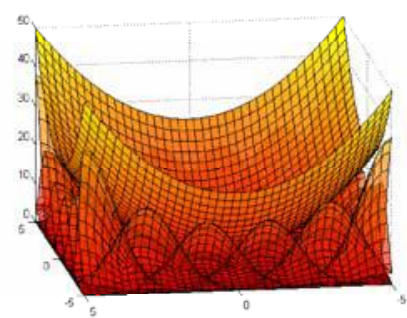

- To be invertible, $\Phi$ must be positive definite. equivalent to having positive eigenvalues. 


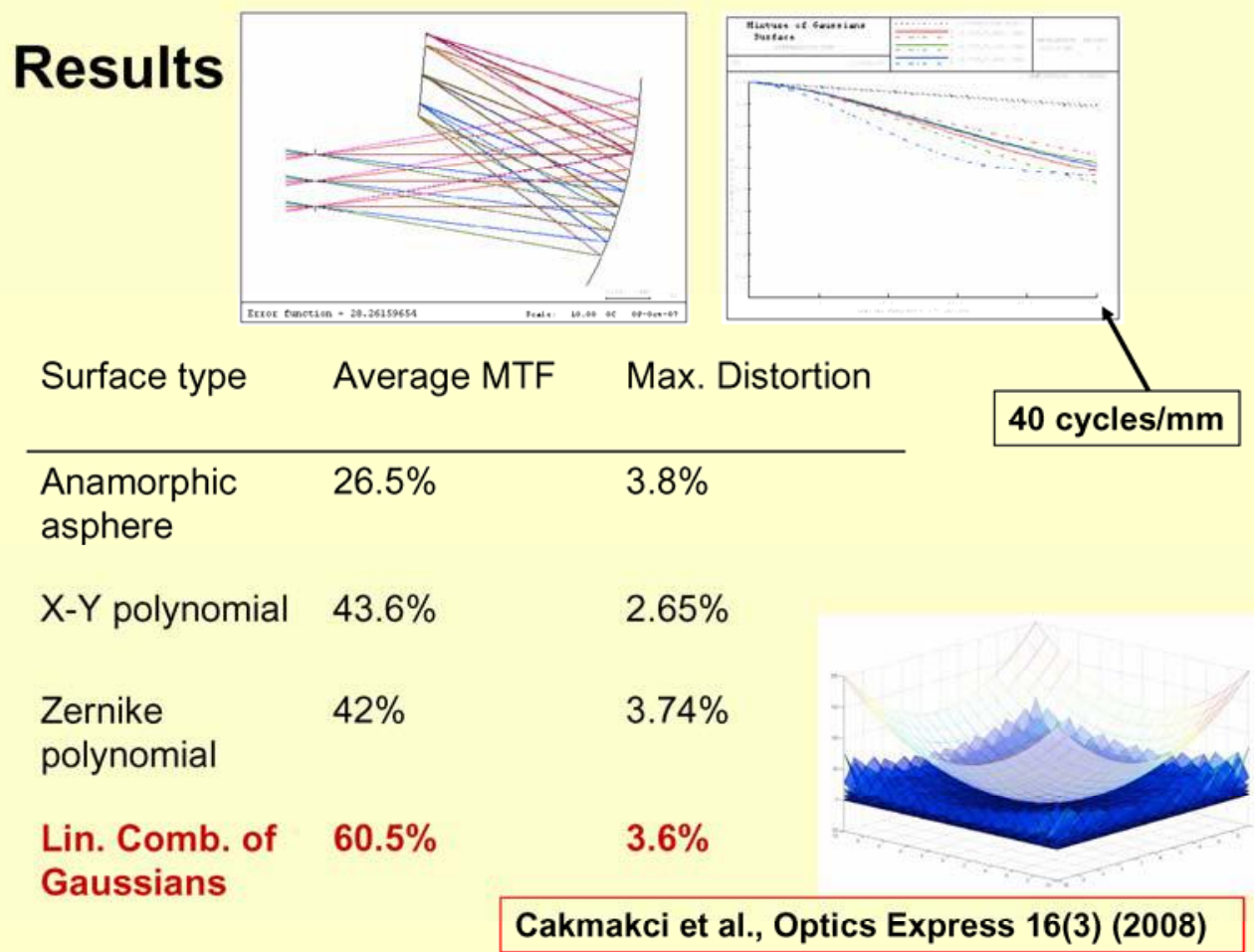

\section{Revisiting the Dual-Element Design: Pupil Size Expansion cakmakci et al. OL (April 2008)}
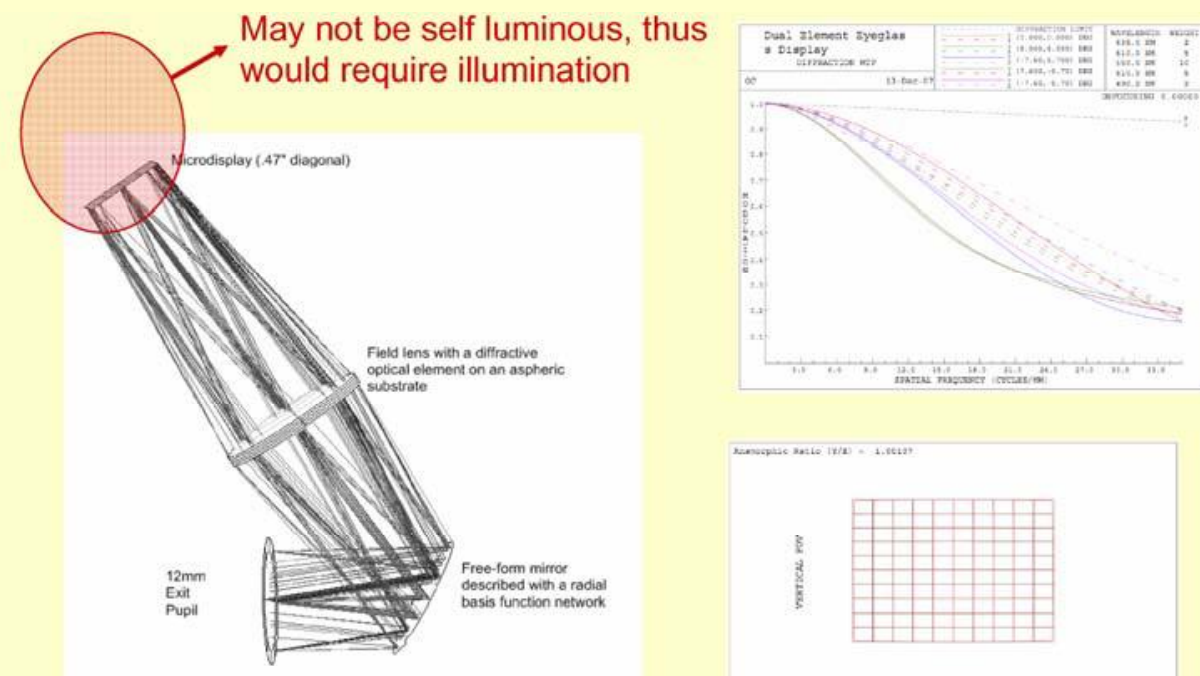

Using a $16 \times 16$ set of basis functions. 


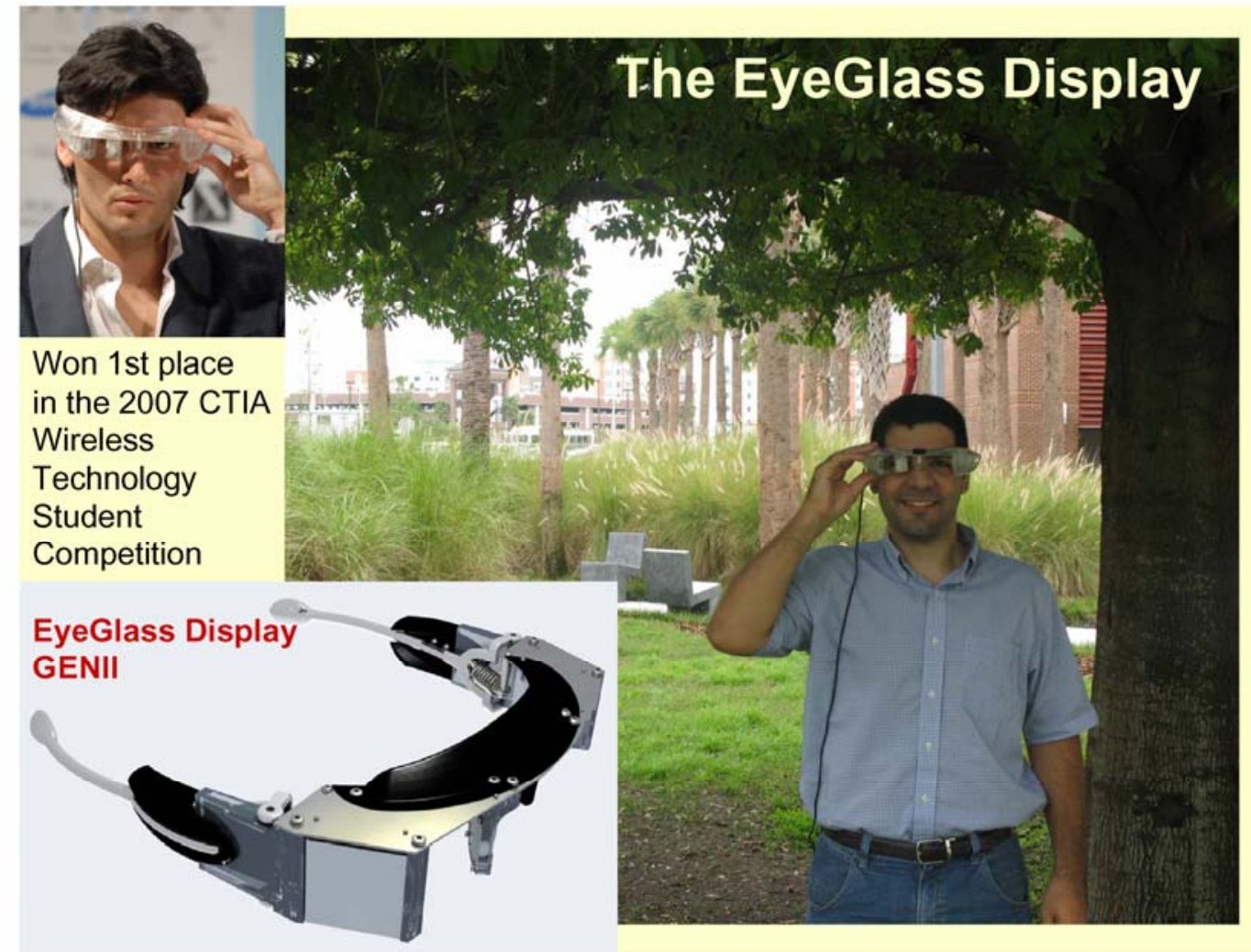

\section{Acknowledgements}

- National Institute of Health (NIH/NLM) First Award (5years)

- National Science Foundation

- EIA 99-86051, IIS/ITR00-82016, IIS/HCI 03-07189

- Office of Naval Research

- N00014-02-1-0261, N00014-02-1-0927, N00014-03-1$0677 \ldots$

- US Army STRICOM, US Army Medical Res., US AirForce

- NASA

- Florida Photonics Center of Excellence

- Industry Partners: METI Corporation, NVIS Corporation, Optical Research Associates 
Downloaded From: https://www.spiedigitallibrary.org/conference-proceedings-of-spie on 26 Apr 2023

Terms of Use: https://www.spiedigitallibrary.org/terms-of-use 